\title{
2. DIE HINTERGRÜNDE \\ DIE VORGESCHICHTE, DER RIFKRIEG \\ UND DIE »RIF-REPUBLIK«
}

\author{
2.1. Marokko als Spielball der europäischen Großmächte \\ bis zum Vorabend des Rifkrieges
}

$\mathrm{Zu}$ Beginn des 20. Jahrhunderts wurde Marokko zum Schauplatz einer Art >Pokerpartier zwischen den hauptbeteiligten europäischen Kolonialmächten Frankreich, Großbritannien, Spanien und Deutschland ${ }^{1}$. Frankreich hatte bereits Algerien erobert (seit 1830) und ein Protektorat in Tunesien (1881) etabliert. Im westlichen Mittelmeer fehlte nur noch Marokko zur Vervollständigung eines nordwestafrikanischen Einfluß- und Herrschaftsbereiches. Spanien hatte sich seit Jahrhunderten punktuell an der marokkanischen Mittelmeerküste festgesetzt. 1497 besetzten spanische Truppen Melilla. 1508 landeten Spanier am Peñon de Vélez de la Gomera (wiederbesetzt 1564). Sie übernahmen 1580 von den Portugiesen Ceuta, und 1673 wurde in der Bucht von Alhucemas auf dem Felsen von Nokour ein Militärposten errichtet. Hinzu kamen 1884 die atlantischen Küstengebiete von Saguía el Hamra und Rio de Oro².

Dennoch, im Vergleich zu Großbritannien und Frankreich, die Ägypten, Algerien und Tunesien kontrollierten, war die Position Spaniens in Nordafrika gegen Ende des 19. Jahrhunderts nur von mäßiger Bedeutung. Wegen der geographischen Nähe Marokkos wollte Spanien an jeglicher Änderung des Status

' HUETZ DE LEMPS, La collaboration, S. 85. Vgl. Helmut NMSCHOWSKI, Das Eindringen in Marokko 1871-1898, in: Helmuth STOECKER (Hg.), Drang nach Afrika. Die deutsche koloniale Expansionspolitik und Herrschaft in Afrika von den Anfängen bis zum Verlust der Kolonien, Berlin 21991, S. 113-120, S. 113 (Marokko als "Zankapfel der Mächte«); Mohammed KENBIB, The Impact of the French Conquest of Algeria on Morocco 1830-1912, in: Hespéris-Tamuda 29 (1991) S. 47-60, S. 52-60. Zum internationalen Hintergrund der Marokkofrage Martin MAYER, Geheime Diplomatie und öffentliche Meinung. Die Parlamente in Frankreich, Deutschland und Großbritannien und die erste Marokkokrise 1904 1906, Diisseldorf 2002 (Beiträge zur Geschichte des Parlamentarismus und der politischen Parteien, 133), S. 17-22, S. 92-115; Moulay A. ALAOUI, Le Maroc face aux convoitises européennes 1831-1912, Salé 2001. Einen guten Gesamtüberblick bieten Charles R. PENNELL, Morocco. From Empire to Independence, Oxford 2003, S. 115-137; VILLALOBOS, El sueño colonial, S. 29-42; José L. VILlanova, El Protectorado de España en Marruecos. Organización política y territorial, Barcelona 2004, S. 29-52.

${ }^{2}$ Charles R. PEnNELL, The Discovery of Morocco's Northern Coast, in: British Journal of Middle Eastern Studies 20 (1993) S. 226-236, S. 229; DerS., A Country, S. 8; MADARIAGA, España y el Rif, S. 37-48; AZIZA, Le Rif, S. 38; AYACHE, Les origines, S. 119 u. S. 119, Anm. 1; LA PORTE, La atracción del imán, S. 29. 
quo beteiligt werden ${ }^{3}$. Dies verstärkte sich noch nach der Niederlage im Spanisch-Amerikanischen Krieg von 1898 und dem daraus resultierenden Verlust von Kuba, Puerto Rico, Guam und den Philippinen. Der Durst nach neuem kolonialen Prestige war gro $\beta^{4}$.

Großbritannien verfolgte in Marokko andere Interessen. Die von einer ausgeprägten "Gallophobie ${ }^{5}$ befallene britische Regierung wollte unter keinen Umständen eine französische Besetzung der marokkanischen Mittelmeerküste zulassen. Die von militärstrategischen Überlegungen geprägte mediterrane Politik Großbritanniens setzte sich zum Ziel, mittels einer Stabilisierung der Kräfteverhältnisse den Seeweg von England nach Indien durch die Straße von Gibraltar und über den Suez-Kanal in Ägypten zu sichern. Die nordmarokkanische Küste - vor allem die Hafenstadt Tanger - und der britische Stützpunkt Gibraltar spielten in dieser Strategie eine erhebliche Rolle ${ }^{6}$. Aus Furcht vor der britischen Reaktion wies Spanien 1902 einen französischen Vorschlag zurück, Marokko untereinander aufzuteilen?

Frankreich und Großbritannien einigten sich am 8. April 1904 auf einen »Entente cordiale« genannten Kompromiß. Frankreich gestand Großbritannien freie Hand in Ägypten zu, während die britische Regierung im Gegenzug - mit Ausnahme von Tanger - auf die politische und militärische Einflußnahme in Marokko verzichtete. Ein geheimer Zusatzvertrag legte in vager Form die Grenzen eines spanischen Teils von Marokko an der Mittelmeerküste fest. Großbritannien plante einen spanischen Puffer zwischen Gibraltar und einem

${ }^{3}$ Carolyn P. BoYD, Praetorian Politics in Liberal Spain, Chapel Hill 1979, S. 22-24; Fleming, Primo de Rivera and Abd-el-Krim, S. 19-22; Villalobos, El sueño colonial, S. 11-15; Eloy MARTín CORRALES, El protectorado español en Marruecos 1912-1956. Una perspectiva histórica, in: Joan NoGUÉ, José L. VillaNOVA (Hg.), España en Marruecos 1912-1956. Discursos geográficos e intervención territorial, Lleida 1999, S. 143-158, S. 146; Pablo LA PORTE, From Cuba to Annual. Spain's Colonial Policy in Morocco and the Crisis of the Liberal System 1898-1923, in: International Journal of Iberian Studies 13 (2000) S. 14-24, S. 14.

${ }^{4}$ BALFOUR, Deadly Embrace, S. 3f.; DERS., Pablo LA PORTE, Spanish Military Cultures and the Moroccan Wars 1909-1936, in: European History Quarterly 30 (2000) S. 307-332, S. 308; CHANDLER, Spain and her Moroccan Protectorate, S. 301; ÁlvAREZ, The Betrothed of Death 2001, S. 1f.; Shannon E. FLemING, North Africa and the Middle East, in: James W. CORTADA (Hg.), Spain in the Twentieth-Century World. Essays on Spanish Diplomacy 1898-1978, London 1980 (Contributions in Political Science, 30), S. 121-154, S. 124; LA PORTE, La atracción del imán, S. 31-36.

${ }^{5}$ MIÈGE, L'arrière-plan diplomatique, S. 224.

${ }^{6}$ FLEMING, Primo de Rivera and Abd-el-Krim, S. 26; MAYER, Geheime Diplomatie, S. 93; AYACHE, Les implications internationales, S. 207; Brian J. MCKERCHER, Esme Howard. A Diplomatic Biography, Cambridge u.a. 1989, S. 235f.

${ }^{7}$ WoOlman, Rebels in the Rif, S. 7; Fleming, Primo de Rivera and Abd-el-Krim, S. 25-27; MAYER, Geheime Diplomatie, S. 134f. 
südlicheren Französisch-Marokko ein ${ }^{8}$. In einer Zusatzklausel des französischspanischen Vertrages vom 3. Oktober 1904 bekam Spanien Territorialrechte für Marokko zugesprochen'. Die italienischen Interessen in Marokko wurden 1902 im Einvernehmen mit Frankreich nach Libyen umgeleitet ${ }^{10}$.

An all diesen Verhandlungen wurde die marokkanische Regierung und deren Verwaltungsapparat nicht beteiligt. Der marokkanische Staat präsentierte sich am Anfang des 20. Jahrhunderts schwach. Unfähigkeit und Korruption kennzeichneten die nach Art der Vetternwirtschaft rekrutierten Beamten. Das Militär war schlecht bewaffnet und disziplinlos. Der tatsächliche Herrschaftsbereich des Sultans beschränkte sich auf Bruchteile von Marokko. Im Landesinneren und besonders in den Gebirgen bestanden die Bevölkerungsgruppen auf ihrer Unabhängigkeit. Die marokkanische Herrscher-Clique benötigte aus Europa Geldanleihen und moderne Militärtechnik und geriet schnell in Abhängigkeiten. Sultan Moulay Abdelaziz interessierte sich ohnehin mehr für Luxusgüter und ein ausschweifendes Hofleben. Weder er noch sein Bruder und Nachfolger Moulay Abdelhafid, der ihn 1908 entthronte, waren in der Lage, die Staatsfinanzen eigenständig zu leiten oder die Ordnung im Lande aufrechtzuerhalten. Es hatte einen vollständigen Kollaps des politischen und finanziellen Systems gegeben. Frankreich gewann mehr und mehr die Oberhand".

${ }^{8}$ ChANDLER, The Responsibilities for Annual, S. 303; Douglas PORCH, The Conquest of Morocco, New York 1983, S. 143; George HILLS, Rock of Contention. A History of Gibraltar, London 1974, S. 389; Michail W. FRUNSE, Die europäischen Zivilisatoren und Marokko, in: DERS. (Hg.), Ausgewählte Schriften, Berlin 1956, S. 47-543, S. 456; AYACHE, Les implications internationales, S. 183; Wolfgang J. MOMMSEN, Das Zeitalter des Imperialismus, Frankfurt a. M. 1969 (Fischer Weltgeschichte, 28), S. 172.

${ }^{9}$ AYACHE, Les implications internationales, S. 184; Jean-Louis MiEGE, Introduction historique. Les relations internationales, in: Abd el-Krim et la République du Rif, S. 25-32, S. 27.

${ }^{10}$ WOOLMAN, Rebels in the Rif, S. 7f.; MIËGE, L'arrière-plan diplomatique, S. 221; Aldo ALBóNICO, L'Italia el il mondo iberico nel primo dopoguerra. Velletia coloniali ed economiche 1919-1923, in: Nuovo rivista storica 66 (1982) S. 82-132, S. 102; Arnold J. TOYNBEE, The Islamic World since the Peace Settlement, London 1927 (Survey of International Affairs $1925,1)$, S. 98.

"LA PORTE, La atracción del imán, S. 37-41; AYACHE, Les origines, S. 25-38; FLEMING, Primo de Rivera and Abd-el-Krim, S. 30; Herbert L. MülLER, Islam, gihâd (»Heiliger Krieg«) und Deutsches Reich. Ein Nachspiel zur wilhelminischen Weltpolitik im Maghreb 1914-1918, Frankfurt a. M. 1991 (Europäische Hochschulschriften, Reihe 3, Geschichte und ihre Hilfswissenschaften, 506), S. 130-134, S. 138f.; Edmund BURKE, Prelude to Protectorate in Morocco. Precolonial Protest and Resistance 1860-1912, Chicago 1976, S. 146-149; Thomas MEYER, „Endlich eine Tat, eine befreiende Tat...«. Alfred von Kiderlen-Waechters »Panthersprung nach Agadir« unter dem Druck der öffentlichen Meinung, Husum 1996 (Historische Studien, 448), S. 156f.; Charles-André JULIEN, Le Maroc face aux impérialismes 1415-1956, Paris 1978, S. 74. 
Im deutschen Reich wurde dies mit wachsender Sorge beobachtet. 1873 hatte mit der Errichtung eines deutschen Konsulats in Tanger der diplomatische Kontakt mit Marokko begonnen. Zunächst hatten Wirtschaftsinteressen im 19. Jahrhundert die Marokkopolitik bestimmt. 1880 gehörte Deutschland zum Kreis des Madrider Vertrages, der den europäischen Unterzeichnerstaaten und den USA den gleichberechtigten und bevorzugten Handel in Marokko zusicherte (»Politik der offenen Tür«). Mit dem deutsch-marokkanischen Handelsvertrag von 1890 faßte die deutsche Wirtschaft endgültig Fuß. Die Propagandisten des Alldeutschen Verbandes sahen vor allem im Westen Marokkos ein mögliches Siedlungsgebiet für Deutsche ${ }^{12}$.

Nach dem Abschluß der »Entente cordiale« zwischen Frankreich und Großbritannien fühlten sich die Deutschen übergangen. Der Tangerbesuch des deutschen Kaisers am 31. Mai 1905 löste die erste Marokkokrise aus. Während der Algeciras-Konferenz im Jahre 1906 berieten 13 Nationen über die wirtschaftliche und politische Zukunft Marokkos. Das Ziel der deutschen Regienung, die Konferenz dafür zu benutzen, die »Entente cordiale« zu sprengen, mißlang. Deutschland wurde isoliert und mußte sich mit einer rein wirtschaftsorientierten Marokkopolitik abfinden ${ }^{13}$. Diese wurde durch das deutsch-französische Marokko-Abkommen vom 9. Februar 1909 vertraglich festgelegt. Deutschland gestand Frankreich ordnungspolitische Aufgaben für Marokko zu, während Frankreich der deutschen Wirtschaft Zusicherungen machte ${ }^{14}$.

${ }^{12}$ MeYer, „Endlich eine Tat«, S. 157; Pierre GuILlEN, Jean-Louis Míge, Les débuts de la politique allemande au Maroc 1870-1877, in: Revue historique 234 (1965) S. 323-352, S. 331; Horst GRÜNDER, Geschichte der deutschen Kolonien, Paderborn u.a. ${ }^{4} 2000$, S. $100 \mathrm{f}$. Vgl. zu den Anfängen der deutsch-marokkanischen Beziehungen im 19. Jahrhundert auch Germain AYACHE, La première amitié germano-marocaine 1885-1894, in: DERS. (Hg.), Études d'histoire marocaine, Rabat 1979, S. 229-247; DERS., La crise des relations germanomarocaines 1894-1897, in: DERS. (Hg.), Études, S. 249-291; Pierre GUILLEN, L'Allemagne et le Maroc 1870-1905, Paris 1967; Francis T. WILlLAMSON, Germany and Morocco before 1905 , Baltimore 1937.

${ }^{13}$ MEYER, «Endlich eine Tat«, S. 160-162; BaLfour, Deadly Embrace, S. 7; Charles R. PEnNELL, Morocco since 1830. A History, London 2000, S. 132f.; BuRKE, Prelude to Protectorate, S. 85-89; Helmuth STOECKER, Helmut NIMSCHOWSKL, Marokko 1898-1914, in: STOECKER (Hg.), Drang nach Afrika, S. 205-222, S. 210-215; MOMMSEN, Das Zeitalter, S. 174f.; Pierre GUILLEN, Les questions coloniales dans les relations franco-allemandes à la veille de la première guerre mondiale, in: Revue historique 248 (1972) S. 87-106, S. 94f.; Jean GaNiAGE, Histoire contemporaine du Maghreb. De 1830 à nos jours, Paris 1994, S. 384-396. Zur internationalen Wirkung des kaiserlichen Tangerbesuchs MAYER, Geheime Diplomatie, S. 154-203.

14 MEYER, "Endlich eine Tat«, S. 165; JULIEN, Le Maroc, S. 81; STOECKER, NiMSCHOWSKI, Marokko, S. 217; MOMMSEN, Das Zeitalter, S. 240. 
Nach der am 1. Juli 1911 durch den "Panthersprung von Agadir ${ }^{15}$ ausgelösten zweiten Marokkokrise einigten sich Frankreich und Deutschland noch im selben Jahr in den Marokko- und Kongo-Verträgen auf eine Ausdehnung der deutschen Kolonialinteressen in Zentralafrika. Frankreich trat Teile von Französisch-Äquatorialafrika gegen den sogenannten 'Entenschnabel ( im Nordosten Kameruns an Deutschland ab. Die deutsche Regierung erkannte dafür die politische und militärische Aktionsfreiheit Frankreichs in Marokko an, verzichtete aber nicht auf wirtschaftliche Betätigung. Damit hatte der ursprüngliche deutsche Wunsch nach einer politischen Gleichberechtigung in Marokko nicht verwirklicht werden können ${ }^{16}$.

Der Weg für Frankreich war frei. In Fes einigten sich der Sultan von Marokko und Frankreich im Vertrag vom 30. März 1912 auf die Errichtung eines französischen Protektorats, das ganz Marokko umfaßte, nicht aber Tanger. Frankreich kontrollierte nun die Außenpolitik, führte innenpolitische Reformen durch und begann mit der "pénétration pacifique» genannten militärischen Durchdringung und Eroberung, letztendlich also mit der Unterwerfung der Bevölkerung des Inlandes. In Rabat errichteten die Franzosen eine Generalresidenz unter Marschall Louis Hubert Lyautey ${ }^{17}$. Spanien erhielt nach dem Abschluß des französisch-spanischen Vertrages vom 27. November 1912 eine eigene Einflußzone (zone d'influence espagnole) im Norden Marokkos, war also de facto eine Art »Untermieter ${ }^{18}$ Frankreichs. Spanien betrachtete das Gebiet als eigenes Protektorat, das zunächst von einer Militärkommandantur und später von einem Hochkommissar in Tetuan geleitet wurde. Damit konnten Großbritannien, Frankreich und Spanien ihre Ziele weitgehend durchsetzen $^{19}$.

Der Ausbruch des Ersten Weltkrieges verdrängte Deutschland mit der Ausweisung oder Internierung aller dort lebenden Deutschen aus Marokko. Dies galt nicht für den spanischen Teil im Norden, denn Spanien verhielt sich neu-

\footnotetext{
${ }^{15}$ Ausführlicher zum "Panthersprung« Emily ONCKEN, Panthersprung nach Agadir. Die deutsche Politik während der zweiten Marokkokrise 1911, Düsseldorf 1981; Jean-Claude AllaIN, Agadir 1911. Une crise impérialiste en Europe pour la conquête du Maroc, Paris 1976; MEYER, „Endlich eine Tat«, S. 211-221; MOMMSEN, Das Zeitalter, S. 241-244; STOECKER, NIMSCHOWSKI, Marokko, S. 218-220.

${ }^{16}$ MEYER, "Endlich eine Tat«, S. 260-264; WOOLMAN, Rebels in the Rif, S. 15; JULIEN, Le Maroc, S. 86; MOMmSEN, Das Zeitalter, S. 246; Fleming, Primo de Rivera and Abd-elKrim, S. 34; GRÜNDER, Geschichte, S. $101 \mathrm{f}$.

${ }^{17}$ PENNELL, A Country, S. 17; TOYNBEE, The Islamic World, S. 99f.

${ }^{18}$ PA-AA Paris 454a, A5141: Botschaft Paris an AA, 13.12.1924.

${ }^{19}$ LA PORTE, La atracción del imán, S. 51-54; PENNELL, Morocco, S. 136-140; Sebastian Balfour, Paul Preston, Introduction. Spain and the Great Powers, in: Dies. (Hg.), Spain and the Great Powers in the Twentieth Century, London 1999, S. 1-12, S. 3; GERSHOVICH, French Military Rule, S. 125; TOYNBEE, The Islamic World, S. 100; JULIEN, Le Maroc, S. 87; PENNELL, A Country, S. 17. In dieser Arbeit werden die Begriffe "Spanisch-Marokko« oder »Spanische Zone Marokkos« benutzt.
} 
tral. Mit der sogenannten »Marokko-Aktion« versuchten die deutschen Militärstrategen, im französischen Protektorat einen Massenaufstand zu entfachen. Das Ziel war, möglichst viele gegnerische Truppen in Marokko zu binden sowie den Zustrom nordafrikanischer Soldaten und die Lieferung von Lebensmitteln nach Frankreich zu verhindern. Der dilettantisch durchgeführte Plan brachte die Franzosen in Marokko nie ernsthaft in Gefahr ${ }^{20}$. Der Vertrag von Versailles besiegelte Deutschlands politischen und wirtschaftlichen Ausschluß aus Tanger und aus dem französischen Protektorat von Marokko. Dessen Bestimmungen fanden jedoch für das spanische Einflußgebiet keine Anwendung. Hier gab es noch spärlichen deutschen Besitz, deutschen Handel und ein deutsches Konsulat in Tetuan ${ }^{21}$.

Für die Tangerfrage, die in den Protektoratsverträgen von 1912 vertagt worden war, stand eine Lösung noch aus. Seit 1892 verwaltete das diplomatische Korps von Tanger die als neutrale Zone betrachtete Stadt und ihre Umgebung. Das gegenseitige Mißtrauen zwischen Großbritannien und Frankreich nach dem Ersten Weltkrieg erschwerte Verhandlungen. Die britische Regierung befürchtete vor allem, daß Tanger von einer verfeindeten Nation - damit meinte man unausgesprochen Frankreich - als Marinestützpunkt gegen Gibraltar und damit gegen den Seeweg nach Indien benutzt werden könnte. Frankreich und Spanien hingegen wollten Tanger in ihre marokkanischen Einflußzonen integrieren. Am 29. Juni 1923 wurde in London eine Tanger-Konferenz mit britischen, spanischen und französischen Delegierten eröffnet. Die Gespräche zogen sich bis zum 18. Dezember 1923 hin, als in Paris das Tanger-Statut unterzeichnet wurde. Es wandelte die Stadt und ihr Umland in eine internationale Zone um. Die Ratifizierung dauerte noch bis zum Mai $1924^{22}$.

${ }^{20}$ KUNZ, MÜLLER, Giftgas, S. 50; Edmund BURKE, Moroccan Resistance, Pan-Islam and German War Strategy 1914-1918, in: Francia 3 (1975) S. 434 464, S. 442. Zur »MarokkoAktion«: MũLLER, Islam, S. 391-412. Zum spanisch-deutschen Verhältnis während des Ersten Weltkrieges: Ramin A. SEPASGOSARIAN, Eine ungetrübte Freundschaft? Deutschland und Spanien 1918-1933, Saarbrücken, Fort Lauderdale 1993 (Forschungen zu Spanien, 12), S. 7-16. Gute Überblicke über die »Marokko-Aktion« bieten folgende Quellen: CADN Maroc DAI 220: Berichte "L'action allemande au Maroc« und »L'action allemande au Maroc (de mars à décembre 1916) « von Commandant Arnaud; CADN Madrid C258: MAE an Ambassade de Madrid, 6.9.1919 mit Marshall Lyauteys Bericht »L'action allemande au Maroc«, 4.7.1919; CADN Tanger Al321: Bericht des französischen Konsulats in Malága »Agissements allemands en zone espagnole de Maroc (du début de la guerre au 15 mars 1917)«; PAAA R21167-21192: »Der Weltkrieg: Unternehmungen und Aufwiegelungen gegen unsere Feinde (Afrikanische Besitzungen Frankreichs)k. Allerdings sind in diesen Akten deutliche Lücken auszumachen, denn ein Großteil wurde direkt nach dem Ersten Weltkrieg vernichtet [Benedikt RÜCHARDT, Deutsch-spanische Beziehungen 1898-1931, München (Diss.) 1988, S. 176].

${ }_{21}$ TOYNBEE, The Islamic World, S. 94, S. 109, Anm. 2; Deutscher Handel in Spanisch-Marokko, in: Tägliche Rundschau, 12.2.1926.

${ }^{22}$ George H. BENNETT, Britain's Relations with France after Versailles. The Problem of Tangier 1919-1923, in: European History Quarterly 24 (1994) S. 53-84, S. 53-55, S. 73-79; 
Regiert wurde die Zone von einem obersten Administrator sowie einem aus dem diplomatischen Korps bestehenden Kontroll-Komitee und einer 26köpfigen legislativen Versammlung, die aus einem Querschnitt der Tangerer Bevölkerung mit deutlich europäischem Übergewicht bestand. Sie wurde vom Vertreter des Sultans, dem Mendoub, geleitet. Für Großbritannien war dabei von wesentlicher Bedeutung, daß die internationale Zone von Tanger sich zu absoluter Neutralität verpflichtete. Keinerlei militärische Einrichtungen waren erlaubt $^{23}$.

\subsection{Das Rif und seine Bevölkerung}

Blättern wir den Kalender zunächst wieder einige Jahre zurück bis an das Ende des Ersten Weltkrieges. Wie sah die Situation in der spanischen Einflußzone Marokkos aus? 62 Kabylen (»Stämme $)^{24}$, die in die Hauptgruppen Senhaja im Osten, Jebala im Westen sowie Ghomara und Rif im Zentralbereich unterteilt wurden, galt es dort zu verwalten. Die Schätzungen für die damaligen indigenen Bevölkerungszahlen reichen von 600000 bis zu einer Million ${ }^{25}$. Schon lange vor der Etablierung der eigenen Einflußzone hatte Spanien von 1900 bis 1909 mit einer weitestgehend kampflosen Besetzung des Gebietes südlich der spanischen Enklave von Melilla begonnen. 1909 bis 1927 folgte die Phase der militärischen Eskalation, begründet durch eine neue Generation von Kolonialoffizieren, den »africanistas«, die eine neue und härtere militäri-

DERS., British Foreign Policy during the Curzon Period 1919-1924, London 1995, S. 141f.; The International Problem of Tangier, in: Foreign Policy Association, Information Service 2 (1926), S. 169-180, S. 169-172; TOYNBEE, The Islamic World, S. 163-174; PENNELL, Morocco since 1830 , S. $195 f$.

${ }^{23}$ Pierre GuILlEN, Aux origines de l'internationalisation de Tanger. Les négociations sur le statut international 1912-1924, in: Abdelouahed BENDAOUD, Mohammed MANiAR (Hg.), Tanger 1800-1956. Contribution à l'histoire recente du Maroc, Rabat 1991, S. 205-215; VAIDON, Tangier, S. 200f.

${ }^{24}$ "Stamm《 - bzw. »tribu« in der französischsprachigen oder "tribe« in der englischsprachigen Literatur - ist die europäisierte Übersetzung des arabischen »Qabila« (»Kabyle«) und meint ebenso eine genealogische Identifikation als größte Einheit, die vom gleichen Vorfahren ab-»stammt《, als auch eine territoriale Identifikation als politische und soziale Einheit, die in den gleichen geographischen Verhältnissen lebt [vgl. PENNELL, A Country, S. 25; DERS., Éxito y fracaso de Abd-el-Krim, in: Historia 1611 (1986) S. 28-36, S. 28; Henry MUNSON, The Mountain-People of Northwestern Morocco. Tribesmen or Peasants, in: Middle Eastern Studies 17 (1981) S. 249-255, S. 250; WOOLMAN, Rebels in the Rif, S. 20 22; HART, Tribe and Society, S. 106; HART, The Aith Waryaghar, S. 8f.].

${ }^{25}$ AYACHE, Les origines, S. 95; MARTín CORRALES, EI protectorado, S. 150; FleMING, Primo de Rivera and Abd-el-Krim, S. 81; BALfour, Deadly Embrace, S. 6. 
sche Gangart propagierten. 1909 bis 1910 verteidigten die Spanier im Raum von Melilla dortige Eisenerzfunde gegen aufständische Rifkabylen. 1911 bis 1912 wurde die Rifrebellion unter Mohammed Ameziane niedergeschlagen ${ }^{26}$.

Nach der Errichtung Spanisch-Marokkos im Jahre 1912 wurde das Gebiet in fünf Territorien eingeteilt: Lucus, Yebala, Gomara, Rif und Quert (nach spanischer Schreibart). Spanische Truppen besetzten im Februar 1913 Tetuan und 1920 Chichaouen. Ansonsten hatten sie militärisch nicht viel erreicht. Der nicht spazifizierte Teil - und damit auch das Rifgebiet im Zentrum SpanischMarokkos - war erheblich größer. Landverbindungen zwischen dem Westen - Larache, Ceuta, Tetuan - und dem im Osten liegenden Melilla gab es nicht $^{27}$. Die einzigen Straßen, die Spanien bis 1924 in seiner marokkanischen Zone baute, verbanden Tetuan mit Tanger und Chichaouen. Letztere war von mangelhafter Qualität und höchstens für militärische Zwecke geeignet ${ }^{28}$. Von einer Entwicklung der Infrastruktur in den wenigen Bereichen des Landesinneren, in denen Spanien tatsächlich das Sagen hatte, war wenig zu merken. Die spanische Verwaltung in Nordmarokko war nicht imstande, wirkliche Verbesserungen für das tägliche Leben der einheimischen Bevölkerung herbeizuführen. Die spanischen Militärs waren wegen ihrer Übergriffe insbesondere in der Rifregion verhaBt ${ }^{29}$.

Das Rif zieht sich - geographisch betrachtet - von der Meerenge von Gibraltar an der gesamten marokkanischen Mittelmeerküste entlang bis zum Moulouya-Fluß im Osten ${ }^{30}$. Es handelt sich um eine zerklüftete Hügel- und Gebirgslandschaft mit Bergspitzen, die im Zentrum die 2 000-Meter-Grenze überschreiten. Nur gelegentlich werden die Berge von meist unfruchtbaren Hochebenen abgelöst. Wenige Flüsse durchziehen die Täler. Nach Osten hin

${ }^{26}$ Gershovich, French Military Rule, S. 125; VillaLobos, El sueño colonial, S. 47-60; Balfour, Deadly Embrace, S. 3-18; BoYD, Praetorian Politics, S. 23f.; FlemIn, Primo de Rivera and Abd-el-Krim, S. 32f.; LA PORTE, From Cuba to Annual, S. 17-21; DERS., La atracción del imán, S. 40-51; CHANDLER, Spain and her Moroccan Protectorate, S. 302; AYACHE, La Guerre du Rif, S. 25-28.

${ }^{27}$ WoOlman, Rebels in the Rif, S. 21, S. 55f.; Stanley G. PAYNe, Politics and the Military in Modern Spain, Stanford 1967, S. 160; Francis KorNER, La Guerre du Rif espagnole vue par la Direction des affaires indigènes française 1912-1924, in: Revue historique 287 (1992) S. 141-156, S. $141 \mathrm{f}$.

${ }^{28}$ Webb MILLER, Ich fand keinen Frieden, Berlin 1938, S. $170 \mathrm{f}$.

${ }^{29}$ Pennel, The Responsibility, S. 68f; Vincent SHEEAN, Abd el Krim and the War in Africa, in: The Atlantic Monthly 136 (1925) S. 251-263, S. 253; PAYNE, Politics, S. 154f; TOYNBEE, The Islamic World, S. 112; BALfOUR, Deadly Embrace, S. 39f.; DerS., LA PORTE, Spanish Military Cultures, S. 324.

${ }^{30}$ HART, Tribe and Society, S. 105; DERS., The Aith Waryaghar, S. 1; AYACHE, Société rifaine, S. 345; KHARChICH, La France, S. 13f.; Joan NoGUÉ, José L. VILlANOVA, La zona norte del Protectorado Español en Marruecos. El marco geográfico, in: DIES. (Hg.), España, S. 101-141, S. 108-111; WoOlman, Rebels in the Rif, S. 20; Ahmed RezzouK, Notes sur l'organisation politique et administrative du Rif, in: Archives marocaines 5 (1905) S. 265275, S. 265. 
nehmen die Regenfälle immer mehr ab, weil die Wolken von der Bergkette des zentralen Rifs aufgehalten werden. Die daraus resultierenden häufigen Dürreperioden gefährden bis heute die kargen Ernten. Der mit einfachen Hilfsmitteln betriebene subsistenzwirtschaftliche Ackerbau gab zu Anfang des 20. Jahrhunderts verschiedene Sorten von Weizen, Früchten und Gemüse her. Bewässerungssysteme oder Dünger waren unbekannt. Vor dem Rifkrieg - so belaufen sich Schätzungen - war im gesamten Rif nur etwa 10-20\% des Gebietes landwirtschaftlich auch durch etwas Viehzucht kultiviert worden, an manchen günstigeren Stellen bis zu 50\%. 1918 bis 1920 gab es in der Region von Alhucemas und in Kebdana wegen einer anhaltenden Dürreperiode Hungersnöte, die Auswanderungsbewegungen nach Tanger und nach Algerien zur Folge hatten ${ }^{31}$.

Das ethnische und sprachliche Zentrum des Rifs wurde im Norden durch die Bucht von Alhucemas und das Mittelmeer sowie durch Snada im Westen, Aknoul im Süden und Midar im Osten begrenzt. Diesem zentralen Rif gehörten die Beqqioua (Bokkoya), Beni (»die Söhne von«) Ouriaghel, Beni Amart, Geznaya, Beni Touzine und Temsamane an, im Westen lebten die Beni Itteft sowie die Beni Bou Ifrah, im Osten die Beni Said, Beni Oulichek, Tafersit, Metalsa, Beni Bou Yahi, Kebdana sowie die fünf Kabylen der Konföderation Guelia $^{32}$. Weit verbreitet waren Aberglauben und Mystik sowie die Heiligenverehrung ${ }^{33}$.

Angaben über die Bevölkerungszahlen zur Zeit des Rifkrieges können allenfalls Schätzungen sein. Germain Ayache beziffert die damalige Gesamtzahl der Rif-Bewohner auf ca. $300000^{34}$, woran die Beni Ouriaghel mit 37000 45000 Menschen den größten Anteil hatten. Es gab keine Städte, allenfalls kleinere Ortschaften oder Dörfer mit zerstreuten Lehmbauten, in denen Wochenmärkte (souks) abgehalten wurden. Diese dienten nicht nur dem Erwerb von Waren, sondern waren auch ein Sammelplatz für politische und soziale Kontakte zwischen den verschiedenen Kabylen ${ }^{35}$. Selbst die spätere Hauptstadt der »Rif-Republik» Ajdir im Gebiet der Beni Ouriaghel war nicht mehr

${ }^{31}$ PenNell, A Country, S. 23f., S. 65, S. 70; Slavin, Anticolonialism, S. 42; Fleming, Primo de Rivera and Abd-el-Krim, S. 58f.; BALFOuR, Deadly Embrace, S. 6; RIVET, Lyautey, Bd. 3, S. 253; AYACHE, Société rifaine, S. 346.

${ }^{32}$ HART, Tribe and Society, S. 105f.; AYACHE, Société rifaine, S. 345; DERS., Les origines, S. 95; Nogué, Villanova, La zona notte, S. 122-127; WoOlman, Rebels in the Rif, S. 21; Günter BARTHEL, Kristina STOCK (Hg.), Lexikon Arabische Welt. Kultur, Lebensweise, Wirtschaft, Politik und Natur im Nahen Osten und Nordafrika, Wiesbaden 1994, S. 499f.

${ }^{33}$ Peter von SIEVERS, Nordafrika, in: Gustave E. von GRUNEBAUM (Hg.), Der Islam, Bd. 2: Die islamischen Reiche nach dem Fall von Konstantinopel, Frankfurt a. M. 1971 (Fischer Weltgeschichte, 15), S. 392-437, S. 397.

${ }^{34}$ AY ACHE, Société rifaine, S. 345; DERS., Les origines, S. 95.

${ }^{35}$ PEnNel., A Country, S. 23f., S. 30, S. 241; HART, The Aith Waryaghar, S. 17; FLEMING, Primo de Rivera and Abd-el-Krim, S. 59; WoOlman, Rebels in the Rif, S. 22. 
als ein größeres Dorf ${ }^{36}$. Die Bevölkerung hatte sich in den Gebirgsregionen auf wenige überhaupt bewohnbare Gebiete zu konzentrieren. In Verbindung mit der Trockenheit führte dies in den Ballungsgebieten häufig zu Blutfehden $^{37}$. Die Beni Ouriaghel galten als besonders tapfere und hartgesottene Kämpfer $^{38}$.

In dieser zerklüfteten und schwer erreichbaren Berglandschaft waren die Menschen über Jahrhunderte praktisch unabhängig geblieben. Sultane hatten in Tanger Gouverneure für das Rif eingesetzt. Sie besaßen aber kaum Einflußmöglichkeiten. Selten erreichten Steuern aus dieser Region die marokkanische Staatskasse. Lediglich als religiöses Oberhaupt wurde der Sultan anerkannt, ansonsten bewahrte die Rifbevölkerung ihre Autonomie ${ }^{39}$. In Europa war bis 1921 praktisch nichts über das Rif bekannt, denn nur sporadisch hatten europäische Besucher das Gebiet bereisen und wieder verlassen können. Christen wurden nicht gerne gesehen. Von daher war der Norden Marokkos für Europa trotz der geographischen Nähe eine Art »Terra incognita« geblieben $^{40}$. Dennoch gab es wegen der algerischen Wanderarbeiter aus dem Rif sowie aufgrund der Handelsbeziehungen nach Tanger, zu den spanischen Enklaven am Mittelmeer und zu Franzosen in Westalgerien bzw. FranzösischMarokko durchaus Kontakte zwischen Rifkabylen und Europäern ${ }^{41}$.

${ }^{36}$ Der Feldzug am Rif, in: Kölnische Zeitung, 9.9.1925; Vincent SHEEAN, My Meeting with Abdel-Krim. The Hieratic, "Semilegendary« Riffi Sultan and his Pirate-Like Gun-Runners, in: Asia 25 (1925) S. 845-857, S. 845; MOWRER, The House of Europe, S. 467; DEGENKOLBE, Erlebnisse, 7.3.1926; LA GARDE, The Moroccan War, 15.9.1923.

${ }^{37}$ AYACHE, Les origines, S. 97; FLEMING, Primo de Rivera and Abd-el-Krim, S. 59; HART, The Aith Waryaghar, S. 4. Beispiele für Blutfehden in: WOOLMAN, Rebels in the Rif, S. 22-27.

${ }^{38}$ SHEEAN, War in Africa, S. 257; Robert Montagne, Abd el Krim, in: Politique étrangère 3 (1947) S. 301-324, S. 302; DERS., Révolution au Maroc, Paris 1953, S. 152; Jean-Louis MIÈGE, Abd el-Krim, in: Encyclopédie berbère, Bd. 1, Aix-en-Provence 1984, S. 73-76, S. 73; Gabriele KRATOCHWIL, Die Berberbewegung in Marokko. Zur Geschichte der Konstruktion einer ethnischen Identität 1912-1997, Berlin 2002 (Islamkundliche Untersuchungen, 247), S. 86, Anm. 221; PENNELL, A Country, S. 25-27.

${ }^{39}$ FLEMING, Primo de Rivera and Abd-el-Krim, S. 58; AYACHE, Les origines, S. 103-105; TOYNBEE, The Islamic World, S. 107; REZZOUK, Notes, S. $268 \mathrm{f}$.

${ }^{40}$ RIVET, Lyautey, Bd. 3, S. 253. Beispiele für europäische Rifreisende im 19. Jahrhundert in: GuILLEN, L'Allemagne, S. 10 u. S. 10, Anm. 7; Jean-Louis MIËGE, Le Maroc et l'Europe 1830-1894, Bd. 4: Vers la Crise, Paris 1963, S. 15, LAZAARE, Marokko, S. 114, S. 125 (Freiherr Heinrich Karl von Maltzan 1858); AZIZA, Le Rif, S. 136; MIËGE, Le Maroc, Bd. 4, S. 48; Frederick V. PARSONS, The Origins of the Morocco Question 1880-1900, London 1976, S. 114; WOOLMAN, Rebels in the Rif, S. 38f; Auguste MoulièrAS, Le Maroc inconnu. Étude géographique et sociologique, Bd. 1: Exploration du Rif, Paris 1895, S. 96-99 (Comte de Chavagnac 1882); PARSONS, The Origins, S. 622 (Henri Duvreyier 1887).

${ }^{41}$ David M. HART, The Saint and the Schoolmaster, or Jbala Warlord and Rifian Reformer Revisited. Conflicting Views of Islam in a Confrontation and Power Clash in Colonial Northern Morocco 1924-25, in: Journal of North African Studies 6 (2001) S. 29-60, S. 39. 


\subsection{Mohammed ben Abdelkrim el Khattabi Vom Kollaborateur zum Gegner Spaniens}

Mohammed ben Abdelkrim el Khattabi, der spätere Begründer der »Rif-Republik«, war den Spaniern gut bekannt. 1881 oder 1882 wurde er in Ajdir geboren. Er gehörte innerhalb der Beni Ouriaghel zur einflußreichen KhattabiFamilie. Sein Vater Mohammed Abdelkrim el Khattabi war Richter (cadi) ${ }^{42}$. Nachdem ihr Haus in Ajdir aufgrund einer Blutfehde niedergebrannt worden war, mußten die Khattabis die Jahre 1892 bis 1898 in Tetuan im Exil verbringen $^{43}$. Dort wuchs in Cadi Mohammed Abdelkrim el Khattabi die Überzeugung, daß nur mit spanischer Hilfe der Fortschritt im Rif Einzug halten könne. Sein zweiter Sohn Si M'hammed wurde der erste Rifkabyle, der eine europäische Ausbildung erfuhr. Er lernte in der Schule der Alliance israélite in Tetuan Französisch ${ }^{44}$, studierte an der Universität von Malaga und wurde in Madrid zum Bergbauingenieur ausgebildet ${ }^{45}$. Der erste Sohn Abdelkrim begab sich nach der traditionellen Schulerziehung in Ajdir und Tetuan an die Universität nach Fes (1902-1904) ${ }^{46}$.

Dieser Aufenthalt sollte der erste Wendepunkt in seinem Leben werden. Neben der üblichen Einweisung in die Grundlagen der arabischen und islamischen Kultur kam Abdelkrim in der politisierten Universitätsstadt mit den Ideen der islam-reformistischen Salafiya-Bewegung in Kontakt. Sie propagierte seit dem Ende des 19. Jahrhunderts vor allem von Ägypten aus die ıErneuerungı des Islam durch eine Rückkehr zu seiner ursprünglichen Vitalität. Die Salafisten wollten der europäischen kolonialen Bedrohung begegnen ${ }^{47}$. Ein

${ }^{42}$ TAHTAH, Pragmatisme 1995, S. 61; HART, The Aith Waryaghar, S. 369; HaRT, The Saint, S. 38f.; LA PORTE, La atracción del imán, S. 99-101; WoOlMAN, Rebels in the Rif, S. 75; Andrés SÁNCHEZ PÉREZ, Abdelkrim, in: Revista de historia militar 17 (1973) S. 123-157, S. 130; PENNELL, Éxito y fracaso, S. 28-36. Einen guten Überblick bieten VILLALOBOS, EI sueño colonial, S. 108-122; CHARQI, Abdelkrim, S. 17-21, S. 29-35, S. 45-63. Die Ähnlichkeit der Namen von Vater und Sobn stiftet manchmal Verwirrung. In dieser Arbeit wird für den späteren Emir des Rifs wegen der besseren Lesbarkeit die Kurzform »Abdelkrim» verwendet. Sein Vater wird zur besseren Unterscheidung "Cadi Mohammed Abdelkrim el Khattabir genannt.

${ }^{43}$ HarT, The Saint, S. 39; FurneauX, Abdel Krim, S. 44; WoOlmaN, Rebels in the Rif, S. 75.

"AYACHE, Les origines, S. 191.

${ }^{45}$ HART, The Aith Waryaghar, S. 371.

${ }^{46}$ AYACHE, Les origines, S. 167; TOYNBEE, The Islamic World, S. 110; WOOLMAN, Rebels in the Rif, S. 75; SHINAR, Abd al-Qadir, S. 163; FURNEAUX, Abdel Krim, S. 45; PENNELL, A Country, S. 49f;; DAOUD, Abdelkrim, S. 49; ABDELKRIM, Memoiren, S. 39f;; MONTAGNE, Abd el Krim, S. 304; DERS., Révolution, S. 155.

${ }^{47}$ HART, The Aith Waryaghar, S. 371f.; DERS., The Saint, S. 40f.; LA PORTE, La atracción del imán, S. 125f. Zur Salafiya-Bewegung: Jamil M. ABUN-NASR, The Salafiyya Movement in Morocco. The Religious Bases of the Moroccan Nationalist Movement, in: St. Anthony 
anderer Student der Universität von Fes, Mohammed Dris ben Saï, beeindruckte Abdelkrim durch seinen strengen arabischen Nationalismus. Er diente ihm später am Anfang des Rifkrieges als Vermittler zum spanischen Geschäftsmann Horacio Echevarrieta ${ }^{48}$.

1906 wurde Abdelkrim von seinem Vater nach Melilla geschickt. Er arbeitete dort als Lehrer und Übersetzer (bis 1913) und wurde Redakteur der spanischen Zeitung »Telegrama del Rif« (1906-1915). Von 1910 an wurde Abdelkrim zusätzlich von der Oficina central de tropas y asuntos indígenas (OCTAI) - dem Spionagedienst für das Militär - beschäftigt. 1914 erfolgte die Ernennung zum Cadi von Melilla. Der junge Abdelkrim war somit als Funktionär der spanischen Administration in Marokko zunächst ein Helfershelfer der Kolonisatoren ${ }^{49}$. Dies kam nicht von ungefähr. Der alte Cadi Mohammed Abdelkrim el Khattabi war seit 1907 ein sogenannter »moro pensionado«, also ein indigener Pensionsempfänger, der den Spaniern Freundschaftsdienste zu leisten hatte ${ }^{50}$. Seine Söhne Abdelkrim und Si M'hammed sollten mit ihren Beschäftigungen im spanischen Milieu europäische Denkweisen und Verwaltungsstrukturen kennenlernen. Die Khattabis sahen in Spanien einen Freund und Verbündeten, der die Beni Ouriaghel aus ihrer ১Rückständigkeit` führen konnte, ohne daß diese dabei ihr autonomes Leben hätten aufgeben müssen. Eine Modernisierung des Landes war ihrer Meinung nach nur mit der Hilfe aus Europa möglich ${ }^{\text {s1 }}$.

Papers 16 (1963) S. 90-105; Reinhard SCHULZE, Islamischer Internationalismus im 20. Jahrhundert. Untersuchungen zur Geschichte der islamischen Weltliga, Leiden u.a. 1990 (Social, Economic, and Political Studies of the Middle East, 41), S. 47f.; Federico S. VIDAL, Religious Brotherhoods in Moroccan Politics, in: Middle East Journal 4 (1950) S. 427-446.

${ }_{48}^{8}$ SHINAR, Abd al-Qadir, S. 163; Kenneth BROWN, Résistance et nationalisme, in: Abd elKrim et la République du Rif, S. 472-477, S. 473f.; TAHTAH, Pragmatisme 1995, S. 146, Anm. 3; WoOlman, Rebels in the Rif, S. 77; HaRT, The Saint, S. 42.

${ }^{49}$ PENNELl, A Country, S. 50; AYACHE, Les origines, S. 169, S. 178, S. 180; SHINAR, Abd al-Qadir, S. 162; MIĖGE, Abd el-Krim, S. 73; SÁNCHEZ PÉREZ, Abdelkrim, S. 135; WoOLMAN, Rebels in the Rif, S. 76; CHTATOU, Rif Oral Tradition, S. 186; Cecil V. USBORNE, The Conquest of Morocco, London 1936, S. 246; ABDELKRIM, Memoiren, S. 43; DAOUD, Abdelkrim, S. 80-84; Carlos SECO SERRANO, La España de Alfonso XIII. El estado y la política 1902-1931, Bd. 1: De los comienzos del reinado a los problemas de la posguerra 19021922, Madrid 1995 (Historia de España, Menéndez Pidal, 38), S. 585; FLEMING, Primo de Rivera and Abd-el-Krim, S. 60; HART, The Aith Waryaghar, S. 372.

${ }^{50}$ MADARIAGA, España y el Rif, S. 398; DIES., Mohammed ben Abdelkrim el Jatabi y las ambivalencias del sprogresoc, in: Fundamentos de antropología 4-5 (1996) S. 14-32, S. 15; HART, The Saint, S. 39; ADMAE Maroc 113, 17: Oficina general de la commandancia general de Melilla an Cadi Mohammed Abdelkrim el Khattabi, 8.12.1912; TAHTAH, Pragmatisme 1995, Dok. 6, S. 135; CADN Maroc DAI 190: Bericht »Notice de renseignements sur Mohamed« o.D.

${ }^{31}$ TAHTAH, Pragmatisme 1995, S. 66f.; ShINAR, Abd al-Qadir, S. 163; PENNell, Morocco since 1830, S. 170; DERS., A Country, S. 50; DERS., "I Wish to Live Peacefully in my Houser. A Moroccan Caid and his Reaction to Colonialism, in: Maghreb Review 6 (1981) S. 49-54, S. 50; AYACHE, Les origines, S. 188f. 
Auf der anderen Seite betrachteten die Spanier die Bucht von Alhucemas und das Gebiet der Beni Ouriaghel als Schlüsselproblem für die Besetzung der eigenen Einflußzone ${ }^{52}$. Dies machte die Khattabis so wichtig. Mit ihrer prospanischen Haltung bildeten sie innerhalb der Beni Ouriaghel allerdings eine Minderheit. Die Fraktion der Gegner von jeglicher Einmischung in die Lebensverhältnisse des Rifs war groß. Als der Vater Abdelkrims und seine Clique der »Freunde Spaniens» Verhandlungen über die Landung spanischer Truppen in der Bucht von Alhucemas führten, wurden ihre Häuser am 6. November 1911 angezündet. Cadi Mohammed Abdelkrim el Khattabi floh zur spanischen Militärfestung nach Nokour und führte anschließend bis zum Mai 1913 ein erneutes Exilantendasein in Tetuan. Mit ausschlaggebend für die heftige Reaktion der Beni Ouriaghel war der Umstand, daB Rifkabylen seit August 1911 unter der Führung von Mohammed Ameziane gegen spanische Truppen rebellierten. Dieser Freiheitskampf, der mit dem Tod Amezianes im Frühjahr 1912 endete, fand ohne die Khattabi-Familie statt ${ }^{53}$.

Während seines Exils verschlechterte sich das Verhältnis von Cadi Mohammed Abdelkrim el Khattabi zu den Spaniern. Er hatte, bevor er 1913 nach Ajdir zurückkehrte, eingesehen, daß die Khattabis ihre Vorstellungen von einem vereinigten Rif als gleichberechtigtem Verhandlungspartner innerhalb der neuen spanischen Einflußzone Marokkos nicht verwirklichen konnten. Dies betraf genauso ihre Geschäfte mit Bergbau-Konzessionen. Der Widerstand Spaniens gegen eine von den deutschen Mannesmann-Brüdern vorgeschlagene wirtschaftliche Chartergesellschaft für Spanisch-Marokko, die der indigenen Bevölkerung weitgehende Autonomie zusagte, tat ein Übriges ${ }^{\text {s4 }}$.

Der Eintritt der Türkei in den Ersten Weltkrieg an die Seite der Mittelmächte im November 1914 wurde zum Schlüsselerlebnis für den Vater Abdelkrims. Es galt, einem islamischen Staat gegen Frankreich beizustehen. Noch im selben Jahr erhielt Cadi Mohammed Abdelkrim el Khattabi den Besuch eines türkischen Agenten. Die Pläne einer Aktion gegen Französisch-Marokko von der neutralen spanischen Einflußzone aus waren ganz in seinem Sinn. Dies war die Möglichkeit, im Rif Prestige zurückzugewinnen ${ }^{55}$. Sein Sohn Abdelkrim wurde während des Ersten Weltkrieges Agent des türkischen Geheimdienstes in Marokko ${ }^{56}$.

Die deutsch-türkische "Marokko-Aktion« sah vor, daß Abdelmalek ben Mohieddine, ein Neffe des legendären algerischen Freiheitskämpfers Abdelkader, versuchen sollte, von Norden aus einen Massenaufstand im französischen Pro-

52 AYACHE, La Guerre du Rif, S. 30.

${ }^{53}$ DERS., Les origines, S. 145-148; WoOLMaN, Rebels in the Rif, S. 44f.

${ }^{54}$ HART, The Saint, S. 42. Siehe hierzu ausführlicher Kapitel 6, S. 216-218.

s5 AYACHE, Les origines, S. 205; MADARIAGA, España y el Rif, S. 405-408; TAHTAH, Pragmatisme 1995, S. 68-70.

${ }^{56}$ BURKE, Moroccan Resistance, S. $458 \mathrm{f}$. 
tektorat zu entfachen ${ }^{57}$. Die Khattabis wurden in der Folgezeit von deutschen Agenten kontaktiert, waren in Waffenschmuggel für Abdelmalek verwickelt und betrieben offene deutsch-türkische Propaganda ${ }^{58}$. Sie waren von der Art und Weise, wie Spanien seine marokkanische Einflußzone bisher geführt hatte, derartig desillusioniert, daß sie aus ihrer kritischen Haltung keinen Hehl mehr machten ${ }^{59}$. Cadi Mohammed Abdelkrim el Khattabi ging Anfang Juni 1915 so weit, gleichgesinnten Rifkabylen nahezulegen, auf ihre spanischen Pensionen zu verzichten. Als Ersatz stellte er deutsche Geldquellen in Aus$\operatorname{sicht}^{60}$.

Mittlerweile war der deutsche Agent Franz Far unter dem amerikanischen Decknamen Elmer B. Lane von Malaga aus ins Rif aufgebrochen, um die Rifkabylen und insbesondere die Beni Ouriaghel dazu zu bewegen, den Aufstand gegen Französisch-Marokko zu unterstützen ${ }^{61}$. Am 25. Juni 1915 traf Far alias Lane in Melilla ein. Mit Abdelkrim besprach er die Weiterreise ins Rif. Mitte September erreichte der deutsche Agent Ajdir ${ }^{62}$. Er versprach Cadi Mohammed Abdelkrim el Khattabi Geld, Waffen und Munition und stellte den Rifkabylen offenbar für die Gegenleistung von $2000 \mathrm{Kämpfern} \mathrm{für} \mathrm{Abdelmalek}$ eine Art Unabhängigkeit nach dem Krieg in Aussicht ${ }^{63}$. Das Lockmittel Franz Fars - die Beibehaltung des autonomen Lebens im Rif mit Unterstützung Deutschlands - hinterließ bei Abdelkrim Spuren. »Der Traum einer Befreiung von der Fremdherrschaft stieg in mir auf«, erinnerte er sich $1927^{64}$.

${ }^{57}$ KUNZ, MÜlLER, Giftgas, S. 50; BURKE, Moroccan Resistance, S. 442, S. 451; GERSHOVICH, French Military Rule, S. 105-107; AYACHE, Les origines, S. 209; MÜLLER, Islam, S. 396; LADREIT DE LACHARRIËRE, Le rêve, S. 99-102.

${ }^{58}$ PENNELL, Morocco since 1830, S. 181; ShINAR, Abd al-Qadir, S. 161, Anm. 99; HART, The Aith Waryaghar, S. 372; MÜLLER, Islam, S. 398; Walter B. HARRIS, France, Spain, and the Rif, London 1927, S. 60; Jacques LADREIT DE LACHARRIÈRE, Abd el Krim, agent et profiteur du bolchévisme, in: Revue des sciences politiques 40 (1925) S. 435-450, S. 445; SÁNChez Pérez, Abdelkrim, S. 137; Payne, Politics, S. 162; FurneauX, Abdel Krim, S. 47.

${ }^{59}$ HART, The Saint, S. 42; SHINAR, Abd al-Qadir, S. 163.

${ }^{50}$ TAHTAH, Pragmatisme 1995, S. 70; AYACHE, Les origines, S. 209; MADARIAGA, España y el Rif, S. 408.

${ }^{61}$ PA-AA R21175, A16900: Generalstab der Armee an AA, 24.5.1915; MÜLLER, Islam, S. 400; USBORNE, The Conquest, S. 225. Germain Ayache und Maria-Rosa de Madariaga liegen mit ihrer Schreibweise »Farle« für »Far» falsch [AYACHE, Les origines, S. 210f.; MADARIAGA, España y el Rif, S. 409].

${ }^{62}$ MÜlLER, Islam, S. 400; TAHTAH, Pragmatisme 1995, S. 70; AYACHE, Les origines, S. 188f.; CADN Tanger A1321: Bericht "Agissements allemands en zone espagnole de Maroc (du début de la guerre au 15 mars 1917)« o.D.; PRO-FO 371, 2415, 156047: Consul General of Barcelona an FO, 18.10.1915; ABDELKRIM, Memoiren, S. 49.

${ }^{63}$ AYACHE, Les origines, S. 210f. u. S. 211, Anm. 40; Otto Graf, Die marokkanische Mauer, Berlin 1933, S. 16; Henri CAMBON, Histoire du Maroc, Paris 1952, S. 275; DAOUD, Abdelkrim, S. 85-87; GrohMANN-KerouACH, Der Siedlungsraum, S. 19, Anm. 4; SÁNCHEZ PÉreZ, Abdelkrim, S. 138; Pierre FonTAINE, Abd el-Krim. Origine de la rebellion nord-africaine, Paris 1958, S. 48.

${ }^{64}$ ABDELKRIM, Memoiren, S. 44. 
Far gelang es jedoch nicht einmal, die Anfänge eines Aufstandes zu organisieren. Als er in seinem Stützpunkt die deutsche Fahne hißte, ließ dies bei denjenigen Rifkabylen, die die deutsche Sache nicht unterstützten, imperiale Absichten Deutschlands vermuten. Am 26. November 1915 starb Far durch eine Vergiftung. Albert Bartels alias Si Hermann wurde sein Nachfolger als Berater Abdelmaleks ${ }^{65}$. Zwar scheiterte die »Marokko-Aktion « letztlich, doch sie bereitete die Basis für den Kampf der Rifkabylen gegen Spanien. Die marokkanischen Widerstandskämpfer standen nicht nur untereinander in ständigem Kontakt, sondern auch mit den Befehlszentren in der Türkei und Deutschland. Sie begannen, politische Loyalitäten in größeren Zusammenhängen zu betrachten. Viele der militärischen Anführer des Rifaufstandes im Jahre 1921 lernten sich während der »Marokko-Aktion« kennen ${ }^{66}$.

Die deutschen Aktivitäten im Norden brachten für Spanien mit sich, daß sich die Gewaltpotentiale der Rifkabylen nach Süden in Richtung FranzösischMarokko verschoben. Zwischen 1914 und 1918 herrschte in der spanischen Zone eine militärische Ruhe wie nie zuvor ${ }^{67}$. Die Khattabis allerdings stifteten aus Sicht der Spanier Unruhe. Im August 1915 veröffentlichte Abdelkrim in der Zeitung »Telegrama del Rif« politische Verlautbarungen, in denen er verlangte, daß sich Spaniens Einfluß auf die Stützpunkte an der Mittelmeerküste beschränken sollte. Seine eigene Hauptaufgabe sah er darin, im Rif eine Regierung zu gründen, um mit Spanien auf gleicher Basis zu verhandeln. Hiermit machte Abdelkrim erstmals auf seine politischen Ambitionen aufmerksam. Die Spanier waren alarmiert. Ein autonomes Rif paßte nicht in ihr Konzept einer Ausweitung des eigenen Herrschaftsbereiches. Wegen der antispanischen Haltung seines Vaters und aufgrund der eigenen antikolonialen Äußerungen wurde Abdelkrim am 6. September 1915 in Melilla verhaftet und als politischer Gefangener interniert. Die Khattabis sollten zur Vernunft gebracht werden ${ }^{68}$.

${ }^{65}$ MŨLlER, Islam, S. 402; Albert BARTELS, Auf eigene Faust. Meine Erlebnisse vor und während des Weltkrieges in Marokko, Leipzig 1925, S. 74f.; USBORNE, The Conquest, S. 226; Sur le front marocain, in: BCAF 26 (1916) S. 71-81, S. 75; Le carnet de Fritz Bottjer, in: BCAF 27 (1917) S. 124-127; GRAF, Die marokkanische Mauer, S. 16; E. KLEINWINTERMANN, Mohamed Abd el-Krim ben Ghattabi. Eine geschichtsphilosophische Studie, in: Zeitschrift für Geopolitik 3 (1926) S. 493-508, S. 500; SÁNCHEZ PÉrEZ, Abdelkrim, S. 138.

${ }^{66}$ BURKe, Moroccan Resistance, S. 472; PENNELl, A Country, S. 54f.

${ }^{67}$ MÜLLER, Islam, S. 393; CHANDLER, Spain and her Moroccan Protectorate, S. 309.

${ }^{68}$ BALFOUR, Deadly Embrace, S. 47f.; TAHTAH, Pragmatisme 1995, S. 71 f.; AYACHE, Les origines, S. 217f.; HART, The Aith Waryaghar, S. 372; FLEMING, Primo de Rivera and Abdel-Krim, S. 62; TOYNBEE, The Islamic World, S. 110f.; SECo SERRANO, La España, S. 586; DAOUD, Abdelkrim, S. 85-87; MADARIAGA, España y el Rif, S. 411-413. 
Der spanische Hochkommissar General Francisco Gómez Jordana schrieb nach Ajdir, daß Abdelkrim als Cadi von Melilla abgesetzt sei ${ }^{69}$. Ein Fluchtversuch am 23. Dezember 1915 endete mit einem Beinbruch, der Abdelkrim für den Rest seines Lebens beim Gehen behinderte. Im August 1916 ließen ihn die Spanier frei, nachdem sein Vater eingelenkt hatte und abermals in Verhandlungen für eine spanische Landung in der Bucht von Alhucemas eingetreten war $^{70} .200000$ Peseten wurden Cadi Mohammed Abdelkrim el Khattabi für den Fall geboten, daß die Spanier in Alhucemas landen könnten, ohne daß ein Schuß falle ${ }^{71}$. Das Versprechen, die Verbindungen mit Abdelmalek aufzugeben, wurde von den Khattabis zunächst nicht eingelöst. Noch im Juli 1916 und im März 1917 schickte Cadi Mohammed Abdelkrim el Khattabi neue Kämpfer zu Abdelmalek, doch dann kam es zum Streit zwischen ihnen, und im Frühjahr 1917 trennten sich ihre Wege ${ }^{72}$.

Erneut waren die Khattabis zwischen die Fronten geraten. Ihre deutschfreundliche Haltung war im Rif nicht überall populär. Abdelkrim war im August 1916 nach seiner Freilassung aus dem Gefängnis nach Ajdir zurückgekehrt. Dort arbeitete er ohne rechte Überzeugung als lokaler Agent für die Spanier und wurde als Ansprechpartner langsam wichtiger als sein Vater. Ein Vabanquespiel - denn die Ressentiments der antispanischen Fraktion innerhalb der Beni Ouriaghel wurden dadurch derartig aufgeheizt, daß die Häuser der Khattabi-Familie und anderer »Freunde Spaniens« im März 1917 erneut zerstört wurden. Spanien nutzte die Gelegenheit, die Beziehungen mit den Khattabis zu verbessern, und setzte Zeichen guten Willens. Spanisches Geld half beim Wiederaufbau. Si M'hammed durfte sein Ingenieurstudium in Madrid aufnehmen, war dort aber auch besser kontrollierbar. Abdelkrim wurde am 15. Mai 1917 wieder als Cadi von Melilla eingesetzt. Doch die nur lau aufgewärmten Beziehungen kühlten rasch wieder ab. Im Dezember 1918 verließ Abdelkrim Melilla für immer. Er folgte dem Ruf seines Vaters, nach Ajdir zurückzukehren, genauso wie sein Bruder Si M'hammed im Januar 1919. Bei den Khattabis hatte sich nach und nach die Einsicht verfestigt, daß Spanien mit der Entwicklung einer - wie sie glaubten, für das Rif nötigen - modernen Infrastruktur überfordert war $^{73}$.

${ }^{69}$ ADMAE Maroc 113, 37: Alto Comisario an Cadi Mohammed Abdelkrim el Khattabi, 3.10.1915; auch in TAHTAH, Pragmatisme 1995, Dok. 7, S. 135 f.

${ }^{70}$ AYACHE, Les origines, S. 221, S. 231; Maria-Rosa de MADARIAGA, L'Espagne et le Rif. Pénétration coloniale et résistances locales 1909-1926, Paris (Diss.) 1987, Bd. 2, S. 97-109; ABDELKRIM, Memoiren, S. 46; MONTAGNe, Révolution, S. 155; DAOUD, Abdelkrim, S. 88f.

${ }^{71}$ ADMAE Maroc 112, 7: Colonel Riquelme an Cadi Mohammed Abdelkrim el Khattabi, 18.6.1916.

7 PENNEll, A Country, S. 53; BARTELS, Auf eigene Faust, S. 126, S. 129, S. 136.

${ }^{73}$ Madariaga, L'Espagne et le Rif, Bd. 2, S. 109-111, S. 113, S. 115f.; PEnNell, A Country, S. 77; TAHTAH, Pragmatisme 1995, S. 76-79; AYACHE, Les origines, S. 242, S. 247; HART, The Saint, S. 43; ABDELKRIM, Memoiren, S. 47f., S. 51. 
Der neue Hochkommissar von Spanisch-Marokko, General Dámaso Berenguer y Fusté, wollte zudem mit Hilfe eines Drei-Punkte-Plans die gesamte Zone militärisch unterwerfen. Das Ziel war, Larache und Ceuta direkt zu verbinden, die Dominanz über den Jebala-Sektor gegen den legendären Ahmed el Risouni (Raisuli) zu erringen und das zentrale Rif zu erobern ${ }^{74}$. Zur Untermauerung der Absichten gründete Spanien 1920 seine eigene Fremdenlegion (»tercio de extranjeros«), die in ihren Anfangsjahren ausschließlich in Marokko eingesetzt wurde ${ }^{75}$.

Hinzu kam, daß der Widerstand im Rif gegen jegliche spanische Einmischung und der Druck auf die »Freunde Spaniens« immer stärker wurden. Die pragmatischen Khattabis sahen sich gezwungen, sich dieser Entwicklung anzuschließen. Sie befanden sich unter den ersten spanischen Pensionsempfängern, die 1919 den Bruch vollzogen. Anfang 1920 setzte sich Cadi Mohammed Abdelkrim el Khattabi an die Spitze der antispanischen Fraktion, um seine eigene Position zu stärken. Im Februar 1920 begaben sich Abdelkrim und sein Onkel Abdeslam ben Mohammed el Khattabi nach Tafersit. Sie wollten mit Hilfe der Temsamane den Widerstand gegen spanische Verbände, die von Osten her vorrückten, organisieren. Plötzlich wurde Cadi Mohammed Abdelkrim el Khattabi krank und mußte im Juli 1920 nach Ajdir zurückgebracht werden. Dort starb er am 7. August 1920. Viele glaubten, er sei auf Veranlassung des spanischen Militärs hin vergiftet worden, aber dies ist bis heute nicht erwiesen ${ }^{76}$.

Der damit verbundene $\mathrm{Haß}$ Abdelkrims auf die spanische Armee übertrug sich nicht auf die Spanier an sich. Abdelkrim und sein Bruder bemühten sich weiterhin um wirtschaftliche Kontakte und bedankten sich ausdrücklich beim Redaktionschef der »Telegrama del Rif«, Cándido Lobera Gizela, für dessen am 11. August 1920 veröffentlichten Nachruf für ihren Vater. Sie gingen in

${ }^{74}$ BOYD, Praetorian Politics, S. 161-165; FlemING, Primo de Rivera and Abd-el-Krim, S. 53-55; Maria-Rosa de MADARLAGA, Le Parti socialiste espagnol et le Parti communiste d'Espagne face à la révolte rifaine, in: Abd el-Krim et la République du Rif, S. 308-366, S. 323; PAYNE, Politics, S. 159; WoOlMAN, Rebels in the Rif, S. 63; CAOM 10 APOM 295: Bericht von Robert Montagne "L'affaire riffaine - Abd el Krim«, 28.5.1937, S. 5.

${ }^{75}$ BALfour, Deadly Embrace, S. 57f; PAYNE, Politics, S. 156; Robert B. ASPREY, War in the Shadows. The Guerilla in History, New York 1994, S. 265; BoYD, Praetorian Politics, S. 172; ÁlvarEZ, The Betrothed of Death 2001, S. 13-25.

${ }^{76}$ AYACHE, Les origines, S. 293, S. 302f.; TAHTAH, Pragmatisme 1995, S. 83; PENNELL, The Responsibility, S. 77; HaRRIS, France, S. 67f.; Jean VILlaIN, Marcel BrUN, Fellah ohne Faruk. Eine Ägyptenreise, Berlin 1958, S. 7. Für die Vergiftungsthese: HART, The Aith Waryaghar, S. 373; DERS., The Saint, S. 43; CHARQI, Abdelkrim, S. 74f.; Jean Wolf, Les secrets du Maroc espagnol. L'épopée d'Abd-el-Khaleq Torres, Casablanca 1994, S. 114; FURNEAUX, Abdel Krim, S. 51f. Auch Abdelkrim verbreitete später, sein Vater sei vergiftet worden [William R. POLK, The Education of a Nationalist. An Interview with Emir Abd elKrim, in: Perspective of the Arab World. An Atlantic Monthly Supplement, New York 1956, S. 24f., S. 25; ABDELKRIM, Memoiren, S. 57]. 
ihrem Brief, der in der Ausgabe vom 22. August 1920 abgedruckt wurde, sogar so weit, vom gemeinsamen »zivilisatorischen « Werk zu sprechen ${ }^{77}$. Hier deutete sich an, daß sich die europäisierten Khattabi-Brüder bereits auf einer höheren Stufe der `Zivilisation ‘ wähnten, die sie in ihrer Heimat noch einzuführen hofften. Sie hatten die Modernisierungsmaßnahmen im Einklang mit Spanien durchführen wollen, doch dem standen die Eroberungsziele des spanischen Militärs im Weg.

Im Oktober 1920 begab sich Abdelkrim an der Spitze von 300 Männern der Beni Ouriaghel ins Gebiet der Temsamane, wo er bei Aït Bou Idhir sein erstes Hauptquartier für den Kampf gegen die Spanier errichtete ${ }^{78}$. Anfang 1921 stieß die spanische Ostarmee von Melilla aus unter ihrem Kommandeur General Manuel Fernández Silvestre bis zur Linie Annual-Tafersit-Azrou-Midar vor. Damit hatte sie mit ihrer seit dem Vorjahr andauernden Offensive das spanisch besetzte Gebiet in Nordmarokko verdoppelt ${ }^{79}$. Silvestre, der eine 25700 Mann starke Armee anführte ${ }^{80}$, war ein klarer Befürworter einer rein militärischen Lösung des spanischen Marokko-Problems und verhöhnte die gegnerischen Rifkabylen wegen ihrer wollenen Umhänge - den Jellabas - als »Petticoat-Soldaten ${ }^{81}$.

\subsection{Das »Desaster von Annual« und seine Folgen}

Der Tod des Vaters war gleichzeitig die Geburtsstunde von Mohammed ben Abdelkrims politischer Karriere im Rif $^{82}$. War er bisher einer von mehreren gleichberechtigten Anführern gewesen, konnte er ab Januar 1921 seine Position ausbauen. Pläne für eine eigene bezahlte Truppe von Soldaten mit einem engen Vertrauten von den Beni Touzine - Mohammed ben Ali el Ouakkili alias Boulahia (»der Bärtige«) - an der Spitze, wurden in die Tat umgesetzt. Die meisten Kämpfer kamen von den Beni Ouriaghel, Beqqioua, Temsamane und Beni Amart. Bis zum Ende des Frühjahres 1921 konnte Abdelkrim mehrere hundert Mann am Jebel el Qama fünf Kilometer westlich von Ouberrane

${ }^{7}$ AYACHE, Les origines, S. 304. Vgl. HERnÁNDEZ MIR, Del desastre a la victoria, Bd. 1, S. 17f.

${ }^{78}$ HART, The Saint, S. 43.

${ }^{79}$ PENNELL, A Country, S. 72; George HILLS, Franco. The Man and his Nation, London 1967, S. 120f;; AYACHE, La Guerre du Rif, S. 60; José L. de MESA GuTIÉRREZ, 1919-1927, casi una década de sangre, in: Antonio CARRASCo GaRCía, Roberto MuÑoz Bolaños (Hg.), Las campañas de Marruecos 1909-1927, Madrid 2001, S. 128-163, S. 134-136.

${ }_{80}$ ÁlvarEZ, The Betrothed of Death 2001, S. 42; BALFOUR, Deadly Embrace, S. 67.

${ }^{81}$ FURNEAUX, Abdel Krim, S. 54.

${ }^{82}$ DAOUD, Abdelkrim, S. 94; WOLF, Les secrets, S. 114. 
zusammenziehen. Ende April 1921 wurde er von einer Versammlung von 50 Scheichs der Beni Ouriaghel und der Beqqioua zu ihrem militärischen Anführer ernannt ${ }^{83}$. Während dieses Treffens sprach er erstmals davon, im Rif einen eigenen Staat mit einer eigenen Regierung und einer eigenen Flagge gründen zu wollen. Das bedeutete eine Umwälzung der bisherigen egalitären politischen Strukturen im Rif hin zu zentralisierten Machtbefugnissen für Abdelkrim $^{84}$. Sein Idealismus mischte sich mit wirtschaftichem Eigeninteresse (Bergbauverträge) und mit persönlichem politischen Ehrgeiz ${ }^{85}$.

Abdelkrim ließ Silvestre mehrmals Warnungen zukommen, nicht weiter ins Rif vorzudringen. Am 1. Juni 1921 überquerte ein kleiner spanischer Stoßtrupp von etwa 250 Mann den Amekrane und besetzte den Ouberrane-Hügel. Die Spanier kamen dem Gebiet der Beni Ouriaghel sehr nahe. Bereits einen Tag später wurde die Anhöhe von den Rifkabylen zurückerobert. Der Rifkrieg hatte begonnen. Abdelkrims Sieg war durch das Überlaufen indigener Kolonialsoldaten erleichtert worden. Lieder wurden über die Schlacht von Ouberrane gesungen. In Scharen strömten neue Kämpfer von den Beni Touzine, Temsamane, Beni Ouriaghel, Beni Amart, Beni Gamil, Beni Bou Ifrah oder Beni Itteft hinzu und ließen die Stärke von Abdelkrims Streitmacht bis Mitte Juni auf $3000-4000$ Mann ansteigen ${ }^{86}$. Für ihn war der aus dem Sieg von Ouberrane resultierende Enthusiasmus für die Ausbildung eines Rifstaates von ausschlaggebender Bedeutung: „Damit begann der Bund der Rifstämme « ${ }^{87}$.

Die Rifkabylen, die sich im bergigen Gelände bestens auskannten, schlugen weiter hart zu. Thren Erfolgen kam die spanische Taktik der Aufsplitterung der Kräfte in Blockhäuser (blocaos) entgegen ${ }^{88}$. Völlig unerwartet wurden das Hauptkontingent des spanischen Expeditionskorps unter General Silvestre und zahlreiche die Truppen begleitende Zivilisten im Stützpunkt Annual von rund 4 000-6 000 Rifkämpfern eingekesselt. Am 21. Juli 1921 begann die Schlacht.

${ }^{83}$ PENNELL, Morocco since 1830, S. 190; POLK, The Education, S. 25; AYACHE, La Guerre du Rif, S. 64; DERS., Les origines, S. 315, S. 321f.; CHARQI, Abdelkrim, S. 81-89; DAOUD, Abdelkrim, S. 97; HART, The Aith Waryaghar, S. 373; WOOLMAN, Rebels in the Rif, S. 80.

${ }^{84}$ PENNELl, A Country, S. 73-75, DERS., Morocco since 1830, S. 190; TAHTAH, Pragmatisme 1995, S. 89.

${ }^{85}$ BOYD, Praetorian Politics, S. 175.

${ }^{86}$ HART, The Aith Waryaghar, S. 374f.; LA PORTE, La atracción del imán, S. 105; ÁlvAREZ, The Betrothed of Death 2001, S. 42-44; BALFOUR, Deadly Embrace, S. 68f.; AYACHE, La Guerre du Rif, S. 85f.; FLEMING, Primo de Rivera and Abd-el-Krim, S. 66f.; SECo SERRANO, La España, S. 592-594; PenNell, A Country, S. 81 f; DeRS., Morocco, S. 190; FuRNEAUX, Abdel Krim, S. 61-63; ChTATOU, Rif Oral Tradition, S. 194f;; HILlS, Franco, S. 121; WOOLMAN, Rebels in the Rif, S. 88; PANDO, Historia secreta, S. 22-25.

${ }^{87}$ ABDELKRIM, Memoiren, S. 66.

${ }^{88}$ ChandLer, The Responsibilities for Annual, S. 70; AYACHE, La Guerre du Rif, S. 109112; BALFOUR, Deadly Embrace, S. 69; ChTATOU, Rif Oral Tradition, S. 196; FleMING, Primo de Rivera and Abd-el-Krim, S. 68f.; HILLS, Franco, S. 122; ABDELKRIM, Memoiren, S. 67f., WOOLMAN, Rebels in the Rif, S. 90; USBORNE, The Conquest, S. $250 f$. 
Silvestre, der seine Gegner unterschätzt hatte, befahl am Tag darauf den Rückzug, der in eine unkontrollierte und panische Flucht ausartete. Die Verluste der Spanier beliefen sich auf über $8000 \mathrm{Mann}$, darunter befand sich auch General Silvestre selbst. Unmengen an militärischem Material fielen in die Hände der Rifkabylen. Das »Desaster von Annual« - eine der größten Niederlagen einer europäischen Kolonialmacht überhaupt - war komplett ${ }^{89}$. Die Unzulänglichkeiten des spanischen Offizierskorps und die schlechte Ausbildung der spanischen Soldaten waren mehr für den Sieg der zahlenmäßig unterlegenen Rifkabylen verantwortlich als deren überlegene Strategie ${ }^{90}$. Abdelkrims Bruder Si M'hammed meinte später, der Sieg von Annual sei ein reines Wunder gewesen ${ }^{91}$.

Die Riftruppen rückten nach Osten vor. Über 130 Militärposten und die spanischen Bergwerke bei Melilla wurden überrannt. Ende August 1921 hatte Spanien das gesamte Gebiet, das seit 1909 in der östlichen Einflußzone erobert worden war, wieder verloren. Die Erfolge festigten Abdelkrims politischen Einfluß im gesamten zentralen Rif. Er war der unumstrittene Anführer ${ }^{92}$. Und

${ }^{89}$ ÁlvarEZ, The Betrothed of Death 2001, S. 44; BALFOUR, Deadly Embrace, S. 52; HART, The Aith Waryaghar, S. 374; CHARQI, Abdelkrim, S. 111-114; DAOUD, Abdelkrim, S. 103f., S. 107; WOOLMAN, Rebels in the Rif, S. 89f.; ABDELKRIM, Memoiren, S. 69-72; USBORNE, The Conquest, S. 251-253. Die Angaben in der Literatur über die Zahl der spanischen Verluste (Tote, Verwundete, Vermißte) von Juli bis August 1921 - offiziell 13192 [HART, The Saint, S. 45] - schwanken erheblich: z.B. ca. 8000 [CHANDLER, The Responsibilities for Annual, S. 68]; über 9000 [LA PORTE, From Cuba to Annual, S. 21]; 8 000-12 000 [BALFOUR, Deadly Embrace, S. 70]; über 10000 [Charles R. PENNELL, Law, Order and the Formation of an >Islamic Residence to European Colonialism. The Rif 1921-1926, in: Revue d'histoire maghrébine 21 (1981) S. 25-39, S. 25; DERS., The Responsibility, S. 67; DERS., Morocco, S. 190]; mindestens 12000 [José E. ÁLVAREZ, Tank Warfare during the Rif Rebellion 1921-1927, in: Armor, Januar-Februar 1997, S. 26-28, S. 26]; 12981 [SECO SERRANO, La España, S. 601]; 13 000-35 000 [KUNZ, MÜLlER, Giftgas, S. 55]; 15000 [Brian CROzIER, Franco. A Biographical History, London 1967, S. 61]; 16000 [HARRIS, France, S. 73]; 18000 [Francis H. MELLOR, Morocco Awakes, London 1939, S. 71]; ca. 19000 [WoOlmaN, Rebels in the Rif, S. 96; GerShOVICH, French Military Rule, S. 124]; 22000 [CHTATOU, Rif Oral Tradition, S. 197]. Zur Bedeutung von Annual: sthe greatest colonial defeat for a European power since the Italian defeat at Adowa at the hands of the Ethiopians on 1 March 1896« [ÁlVAREZ, The Betrothed of Death 2001, S. 63, Anm. 16; vgl. FLEMING, Primo de Rivera and Abd-el-Krim, S. 70]; sthe worst colonial setback suffered by a European nation since the Italians were routed by the Abessinians at Adowan [HOISINGTON, Lyautey, S. 185]; "the worst disaster that had ever befallen any colonial power anywhere in a sbrush-fires war « [HART, The Aith Waryaghar, S. 374]; sone of the most severe colonial defeats of this century « [CHANDLER, The Responsibilities for Annual, S. 68]; "main defeat of the Spanish army in Morocco « [LA PORTE, From Cuba to Annual, S. 21]; wthe worst defeat of a colonial army in Africa in the twentieth century « [PENNELL, Morocco since 1830, S. 190].

${ }^{90}$ KUNZ, MÜLLER, Giftgas, S. 56; MELLOR, Morocco, S. 66-68.

${ }^{91}$ FURNEAUX, Abdel Krim, S. 70f.

92 Payne, Politics, S. 169; TOYNBEe, The Islamic World, S. 116; ÁlvareZ, The Betrothed of Death 2001, S. 47; PENNELL, A Country, S. 86, S. 91; DRIESSEN, Images of Spanish 
Abdelkrim hatte Glück. Die beste Ernte seit 14 Jahren verschaffte eine Nahrungsreserve, die drei Jahre ausreichen sollte. Abdelkrim verbot unter strengsten Strafen jeglichen Export von Lebensmitteln ${ }^{93}$.

Melilla lag, nachdem Silvestres Armee aufgerieben worden war, praktisch schutzlos vor den Riftruppen. Eilig wurden Kontingente der spanischen Fremdenlegion von Tetuan nach Melilla verlegt. Nun standen Ende Juli 1921 wieder 14000 Mann in der Stadt ${ }^{94}$. Nach all seinen Erfolgen beging Abdelkrim einen militärstrategischen Fehler. Er ließ seine Truppen vor Melilla halten, weil er unter anderem befürchtete, andere europäische Nationen würden zum Schutz ihrer dort lebenden Staatsbürger intervenieren ${ }^{95}$. Damit behielten die spanischen Truppen ihren größten Stützpunkt in der östlichen Einflußzone. Im Nachhinein ärgerte sich Abdelkrim: „Diesen Befehl bereue ich bitter. Es war mein größter Fehler. [...] Die ganze spätere Entwicklung der Dinge war die Folge dieses Fehlers ${ }^{96}$.

So begann am 12. September 1921 von Melilla aus der spanische Gegenstoß. Da viele Rifkämpfer zur Ernte wieder in ihre Dörfer zurückgekehrt waren, drang die spanische Ostarmee bis zum Januar 1922 ohne sonderlichen Widerstand bis Dar Driouch vor. Ende 1922 hatten die Spanier fast wieder die Frontlinie vom Juni 1921 erreicht ${ }^{97}$. Abdelkrims Kontingente hatten die militärische Lage jedoch unter Kontrolle und schafften es nach einer einwöchigen Gegenattacke im März 1922, eine militärische Pattsituation aufrechtzuerhalten $^{98}$. In langen Verhandlungen mit Abdelkrim versuchte die spanische Regierung, die Gefangenen der Kämpfe von Annual und Jebel Aroui freizubekommen. Schließlich überbrachte der baskische Millionär Horacio Echevarrieta im Januar 1923 ein Lösegeld in Höhe von 4270000 Peseten $^{99}$.

Colonialism, S. 62; BOYD, Praetorian Politics, S. 176-182; CHANDLER, The Responsibilities for Annual, S. 71; DERS., Spain and her Moroccan Protectorate, S. 312; HLLS, Franco, S. 124; Slavin, Anticolonialism, S. 45f.; AYACHE, La Guetre du Rif, S. 141-158; DaOud, Abdelkrim, S. 108; WOOLMAN, Rebels in the Rif, S. 92-94.

${ }^{93}$ PRO-FO 371, 7066, 184, 197: Consul of Tetuan an CG Tangier, 29.4.1921; PRO-FO 371, 7082, 8256, 170: John Arnall an FO, 13.8.1921.

94 ÁlVAREZ, The Betrothed of Death 2001, S. 46; PENNELl, A Country, S. 85f.

${ }^{95}$ GerSHOVICH, French Military Rule, S. 124f.; KHARCHICH, Observations, S. 230; Moulay A. ALAOUI, Le Maroc du traité de Fès à la liberation 1912-1956, Rabat 1994, S. 45; KUNZ, MǗLLER, Giftgas, S. 55.

${ }^{96}$ ABDELKRIM, Memoiren, S. 74.

${ }^{97}$ PenNell, A Country, S. 98; Álvarez, The Betrothed of Death 2001, S. 75-77; FLEMING, Primo de Rivera and Abd-el-Krim, S. 71; PAYNE, Politics, S. 181; HILls, Franco, S. 130; WOOLMAN, Rebels in the Rif, S. 103-105.

${ }^{98}$ Gershovich, French Military Rule, S. 125; PennelL, A Country, S. 105; FuRnEauX, Abdel Krim, S. 93.

${ }_{99}$ Pablo DtAZ MORLÁN, Horacio Echevarrieta 1870-1963. El capitalista republicano, Madrid 1999, S. 134f.; Carlos SECO SERRANO, El plano inclinado hacia la dictadura 1922-1923, in: José M. JOVER ZAMORA (Hg.), La España de Alfonso XIII. El estado y la política $1902-$ 1931, Bd. 2: Del plano inclinado hacia la dictadura al final de la monarquía 1922-1931, Ma- 


\subsection{Die Proklamation der »Rif-Republik»}

Im Juni 1922 hatte Abdelkrim die Bevölkerung des Rifs informiert, daß er eine unabhängige Republik mit eigener Regierung und eigener Armee in Planung habe ${ }^{100}$. Am 18. und 22. Januar sowie am 1. Februar 1923 sicherten die wichtigsten Anführer innerhalb des Rifs Abdelkrim in politischen Erklärungen ihre Loyalität zu. Sie ernannten ihn in religiösen und politischen Termini zu ihrem Befehlshaber. Der 1. Februar 1923 war auch das Proklamationsdatum der »Rif-Republik ${ }^{101}$. Die Gründung des Rifstaates ist im Zusammenhang mit ähnlichen Entwicklungen in anderen islamischen Ländern zu betrachten. 1918 hatte eine "aserbeidschanische Republik" bestanden, 1918 bis 1920 eine "tripolitanische Republik«. In Syrien, im Irak und in Palästina gab es nationalistische Bewegungen. Mustafa Kemal errichtete 1923 in der Türkei eine Republik $^{102}$.

Abdelkrim und sein Bruder Si M'hammed gaben ihrem Staat die Bezeichnung »Republik« (ad-dawla al-jumhuriya ar-Riffiya), um nach außen aufzuzeigen, daß mehrere Kabylen in einer Allianz lebten ${ }^{103}$. Im Rif selbst verwendete man die Formulierung "Rif-Front« (al-jibha ar-Riffiya). Dies brachte das

drid 1995 (Historia de España, Menéndez Pidal, 38), S. 11-130, S. 65-69; José M. MARÍN ARCE, El gobierno de la concentración liberal. El rescate de prisioneros en poder de Abdel-Krim, in: Revista de la Facultad de geografia e historia 1 (1987) S. 163-181, S. 167, S. 176f.; HART, The Aith Waryaghar, S. 376; WOOLMAN, Rebels in the Rif, S. 111; BOYD, Praetorian Politics, S. 239-241; ÁlVAREZ, The Betrothed of Death 2001, S. 85; SLAVIN, Anticolonialism, S. 48; SHAT 3H134: Ambassade de Madrid an MG, 2.2.1923; PA-AA R72042, L057452: Botschaft Madrid an AA, 28.1.1923; PRO-FO 371, 9473, 898, 52: Embassy of Madrid an FO, 31.1.1923. Zur Lage der spanischen Gefangenen im Rif: Jaime M. de GANTE, El cautiverio en Axdir. La vida de los prisioneros, in: Blanco y Negro, 8.1.1922; Alfredo BosQue Coma, Prisionero de Abd el-Krim, in: Historia 1616 (1992) S. 23-28.

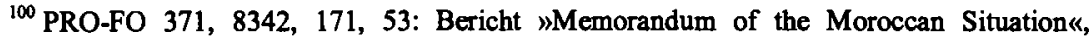
5.7.1922.

${ }^{101}$ ChTatou, Aspectos, S. 66f.; PAYNe, Politics, S. 185; Daniel RIvet, Le Maroc de Lyautey à Mohammed V. Le double visage du protectorat, Paris 1999, S. 355. Der Text der Erklärung vom 1. Februar 1923 ist in englischer Übersetzung abgedruckt in: PENNELL, A Country, App. 3, S. 248-251. Andere Autoren datieren - basierend auf einem Übersetzungsfehler von Abdelkrims >Memoirenschreiber< Jacques Roger-Mathieu [ABDELKRIM, Memoiren, S. 75] - das Gründungsdatum der "Rif-Republik « fälschlicherweise auf den 1. Februar 1922 [z.B. Jamil M. ABUN-NASR, A History of the Maghrib, Cambridge u.a. ${ }^{2} 1975$, S. 365; RIVET, Lyautey, Bd. 3, S. 260, Anm. 481; PAYNE, Politics, S. 178; MADARIAGA, España y el Rif, S. 555; KRATOCHWIL, Die Berberbewegung, S. 87; Josep L. MATEO DIESTE, La »hermandad« hispano-marroquí. Política y religión bajo el Protectorado español en Marruecos 1912-1956, Barcelona 2003, S. 153].

${ }_{102}$ Reinhard SCHULZE, Geschichte der islamischen Welt im 20. Jahrhundert. München 1994, S. 83-85; KHARCHICH, La France, S. 7.

${ }^{103}$ PENNELL, A Country, S. 133f., S. 257. 
gegen die Invasoren gerichtete Bündnis eher zum Ausdruck ${ }^{104}$. Öffentliche Ausrufer auf den Wochenmärkten oder die Imame der Moscheen verkündeten Abdelkrims Anordnungen. In Gedichten und Gesängen wurde der Emir des Rifs gepriesen ${ }^{105}$. Der Rifstaat, in dem nur Abdelkrim per Gesetzesdekret (dahir) das Sagen hatte, trug autokratische bis diktatorische Züge. Alle Macht war in Ajdir konzentriert. Dort herrschte eine "straff organisierte Zentralgewalt ${ }^{106}$, denn Abdelkrim mußte gegen Widerstände angehen und traditionelle tribale Partikularismen zu einer Gesamtheit zusammenfügen ${ }^{107}$. Nur in dieser, die bisherigen Blutfehden überbrückenden Einheit sah er die wahre Kraft der in zahlreiche Kabylen zersplitterten Rifbevölkerung ${ }^{108}$.

Zu keinem Zeitpunkt hat sich Abdelkrim selbst als Sultan bezeichnet, wie Zeitzeugen häufiger behauptet haben ${ }^{109}$. Möglicherweise handelte es sich um einen Übersetzungsfehler, den vor allem die französische Propaganda gern aufgriff. Europäer, die Abdelkrim im Rif zu Gesicht bekamen, waren zum Teil überrascht, einen recht kleinen, ruhigen Mann mit festem Blick, der schlichte landestypische Kleidung trug, kennenzulernen ${ }^{110}$. Genauso war Abdelkrims Regierungssitz in Ajdir ein karges Lehmgebäude, das den von orientalistischen Stereotypen geprägten ausländischen Besuchern, die eigentlich einen Palast erwartet hatten, gelegentlich Anlaß zur Enttäuschung gab ${ }^{111}$.

${ }^{104}$ KHARCHICH, La France, S. 119. Vgl. Larty RUE, Tribune Gets Peace Terms of Riffians, in: Chicago Tribune, 23.6.1925.

${ }^{105}$ Pennell, A Country, S. 129, S. 143; TAHTAH, Pragmatisme 1995, S. 91; ShINAR, Abd al-Qadir, S. 164f.; YoussoufI, Les institutions, S. 93; ChTATOU, Aspectos, S. 68; DERS., Rif Oral Tradition, S. 193, S. 197, S. 199.

${ }^{106}$ NIMSCHOWSKI, Grundzüge, S. 267.

${ }^{107}$ KRATOCHWIL, Die Berberbewegung, S. 88; MATEO DiESTE, La »hermandad«, S. 153.

${ }^{108}$ Nathalie LOUTSKAIIA, À propos de la structure intérieure de la République du Rif, Moskau 1960, S. 10 mit Bezug auf ein Interview mit Abdelkrim.

${ }^{109}$ HART, The Aith Waryaghar, S. 377; RUE, Tribune, 23.6.1925. Beispiele: Léon GABRIELLI, Abd-el-Krim et les événements du Rif 1924-1926, Casablanca 1953, S. 19; BEVAN, Spain and Morocco II, 23.10.1923; Vincent SHEEAN, Abd-el-Krim's Terms Get Out of Morocco, in: Chicago Tribune, 7.2.1925; DERS., Riff General Gives Views on de Rivera, in: Chicago Tribune, 14.2.1925; LA GARDE, The Rif Rising, 25.8.1923; MEYER, Dreitausend Kilometer, S. 116; SPENCER PRYSE, Through the Lines, S. 627; Hans A. LANGLET, Life in the Riff, in: Manchester Guardian, 1.5.1926; MOWRER, The House of Europe, S. 468 mit Bezug auf Aussagen des deutschen Deserteurs Josef Klems.

${ }^{110}$ Z.B. DU TAILlIS, Behind the Moorish Lines, S. 168; BEVAN, Spain and Morocco II, 23.10.1923; LA GARDE, The Moroccan War, 15.9.1923; WARD PRICE, Extra-Special Correspondent, S. 170; Mọrer, The House of Europe, S. 475f.; SHEEAN, Abd-el-Krim's Terms, 7.2.1925; RUE, News, S. 275f.; Hans A. LANGLET, Abdel Krim's Views on a Riff Peace, in: Manchester Guardian, 29.4.1926.

${ }^{11}$ Z.B. DegenKolBe, Erlebnisse, 7.3.1926; WARD PrICE, Extra-Special Correspondent, S. 168; MOWRER, The House of Europe, S. 467. 
Familienmitglieder, Verwandte und Freunde, die vomehmlich aus Ajdir stammten, besetzten die Schlüsselpositionen der Regierung. Abdelkrim stand als Emir an der Spitze, sein Bruder Si M'hammed war sein Stellvertreter und zugleich Oberbefehlshaber der Rifarmee, sein Schwager Si Mohammed Azerkane Außenminister, sein Onkel Si Moulay Abdeslam ben Mohammed el Khattabi Finanzminister. Die Versammlung von Repräsentanten der Rif-Kabylen hatte lediglich beratende Funktionen ${ }^{112}$. Daneben gab es eine Reihe weiterer Posten wie z.B. Steuereintreiber, Protokollchef, Leiter der Leibwache, Marineinspektor oder Zahlmeister der regulären Riftruppen. Repräsentanten der Rifregierung befanden sich in Algerien (lange Zeit Caïd Haddou ben Hammou), Französisch-Marokko (Si M'hammed ben Mohammed el Hassan el Ouezzani), Tanger (Si Abdelkrim ben el Haj Ali Luh, Si Larbi el Ouezzani und Moulay Ali el Khamlichi) und Paris (Ahmed Balloul). Si Mohammed Boujibar war Experte für Großbritannien ${ }^{113}$. Im Außenministerium arbeitete der algerische Journalist Si el Hassan ben Abdelaziz Tlemcani ${ }^{114}$. Stolz trugen der Lufffahrtminister Caïd Haddou ben Hammou ${ }^{15}$ und der $» B e r a t e r$ der Delegation der Rif-Republik zu auswärtigen Staaten« Si Mohammed Boujibar ihre Visitenkarten mit sich ${ }^{116}$. Die Spitzenfunktionäre waren durchweg eher jung. Nur Abdelkrims Onkel Abdeslam war fast 50 Jahre alt ${ }^{17}$.

Sie repräsentierten eine neue seuropäisiertere، Generation im Rif, die sehr viel aufgeschlossener war als die ihrer Vorfahren. Abdelkrim und sein Bruder Si M'hammed waren in europäische Schulen gegangen. Si Mohammed Boujibar und Si Mohammed Mohammedine Hitmi - beide mit Abdelkrim verschwägert - sprachen Französisch und Englisch. Da sie den westlichen Lebensstil kannten, waren sie bestens als Gesandte für Paris oder London geeignet ${ }^{118}$. Caïd Haddou ben Hammou von den Beqqioua hatte in Algerien eine französische Ausbildung erfahren, die ihm nun im Rif zugute kam. Andere

${ }^{112}$ Chtatou, Aspectos, S. 67; TAHTAH, Pragmatisme 1995, S. 91-94; MADARIAGA, España y el Rif, S. 568f.; PENNELl, A Country, S. 55, S. 126-128; DerS., Morocco, S. 193; KHARCHICH, La France, S. 92-94; HART, The Aith Waryaghar, S. 371, S. 377-380; GABRIELLI, Abd-el-Krim, S. 42-44; Goded Llopis, Marruecos, S. 84f.; SAlas LaRrazábal, Protectorado, S. 147f.; WoOLMAN, Rebels in the Rif, S. 146-148. Der Begriff »Nationalversammlung" trifft den Sachverhalt nicht [YOUSSOUFI, Les institutions, S. 87; NIMSCHOWSKI, Grundzäge, S. 266].

${ }^{113}$ CADN Tanger B30: RG Rabat an CG Tanger, 26.9.1924; CADN Maroc DAI 193: Tabor de police $\mathrm{N}^{\circ} 1$, Note, 25.11.1924.

114 FuRNeauX, Abdel Krim, S. 157; WoOlmaN, Rebels in the Rif, S. 147.

115 „Ministre de l'aviation, agent politique du Ministère des affaires étrangères riffaines" [FURNEAUX, Abdel Krim, S. 72].

${ }^{116} \gg$ M. Boudjibar, Councillor of the Mission from the Riffian Republic to Foreign Powers [LANGLET, Life, 1.5.1926].

${ }^{117}$ HART, The Aith Waryaghar, S. 380.

${ }^{118}$ GABRIELli, Abd-el-Krim, S. 44f.; Charles A.P. GARDINER, I Become Ambassador to Riffs, in: Illustrated Sunday Herald, 26.7.1925; SPENCER PRYSE, Through the Lines, S. $641 \mathrm{f}$. 
waren durch Geschäftsbeziehungen, Schmugglertätigkeiten oder Militärdienst mit der europäischen Lebensart vertraut. Auf diese Weise gelangten Männer wie zum Beispiel Raïs Messaoud Amar alias Sibbera - der Hauptverantwortliche für die kleine Flotte von Rifschiffen - an wichtige Positionen ${ }^{119}$.

Die Staatsflagge mit einem grünen Halbmond und einem grünen sechszackigen Stern vor rotem Hintergrund ähnelte der türkischen Fahne. Sie wehte in der Hauptstadt Ajdir und an allen Fronten als Zeichen für einen unter dem Banner des Islam stehenden Staat. Eine eigene Landeshymne wurde gesun$\operatorname{gen}^{120}$. Der Rifstaat hatte höchstens eine vage nationalistische Ideólogie, die die Bewohner des Rifs verinnerlichen sollten. Eine Nation war mehr Wunschgedanke denn Realität ${ }^{121}$. Die "Rif-Republik» stellte trotzdem den ersten erfolgreichen Versuch dar, eine zentralisierte politische Ordnung im Norden Marokkos zu errichten ${ }^{122}$. Die Anfänge einer staatlichen Organisation und Verwaltungsstruktur auf der Grundlage islamischen Rechts wurden in den wenigen Jahren des Bestehens der "Rif-Republik» aufgebaut. Die Scharia diente gleichzeitig als Legitimation für das neue Regime und als neue Sozialordnung, die das alte System der Konfliktregulierung durch Blutfehden ablösen sollte und berberische religiöse Gebräuche mit dem Blick auf die Organisation eines modernen Staates abzuschaffen versuchte. Die Sklaverei sowie der Handel und Konsum von Haschisch wurden verboten, die Korruption unter strenge Strafe gestellt ${ }^{123}$. Hiermit verwirklichte Abdelkrim einige seiner salafistischen Ideale.

\section{${ }^{119}$ PENNELL, A Country, S. 55f., S. 100.}

${ }^{120}$ Ibid. S. 133; DERS., The Rif War, S. 235; HoISINGTON, Lyautey, S. 192; GODED LLOPIS, Marruecos, S. 95; ChTATOU, Rif Oral Tradition, S. 201.

${ }_{121}$ AYACHE, Société rifaine, S. 369; WoOLMAN, Rebels in the Rif, S. 76; PENNELL, The Rif War, S. 245. Den nationalen Charakter des Rifstaates heben hervor: SHINAR, Abd al-Qadir, S. 165; BOYD, Praetorian Politics, S. 175; Shannon E. FLEMING, Primo de Rivera and Spain's Moroccan Problem 1923-1927, in: Journal of Contemporary History 12 (1977) S. 85-99, S. 85; LOUTSKAİA, À propos, S. 9. Weitere Beispiele aus der arabischen Welt, die Abdelkrim als Vorkämpfer eines marokkanischen Nationalismus sehen, in: TAHTAH, Pragmatisme 1995, S. 3f., S. 31-48; LA PORTE, La atracción del imán, S. 123-125. Völlig falsch liegt Reinhard Schulze, der Abdelkrims "Rif-Republik« als Versuch sieht, »einen unabhängigen marokkanischen Staat« und »eine neue, nicht-sultanische Ordnung des Landes« herbeizuführen [SCHULZE, Geschichte, S. 86]. Für eine derartige Behauptung gibt es keinerlei Beweise [PENNELl, Morocco, S. 146].

${ }^{122}$ FLEMNG, Primo de Rivera and Abd-el-Krim, S. 218, S. 239; HoISINGTON, Lyautey, S. 188; KHARCHICH, La France, S. 91; Jean-Paul ChARNAY, Technique et géosociologie. La Guerre du Rif. Le nucléaire en Orient, Paris 1984 (Travaux du centre d'études et de recherches sur les stratégies et les conflits, Série contemporaine, 4), S. 21; SHINAR, Abd al-Qadir, S. 164; ABUN-NASR, A History, S. 365; RIVET, Le Maroc, S. 355; TAHTAH, Pragmatisme 1995, S. 94.

${ }^{123}$ PeNnell, A Country, S. 126f., S. 144, S. 146; MATEO DieSTE, La whermandad«, S. 350353; KRATOChwIL, Die Berberbewegung, S. 89; Vincent ShEEAN, Riffis Fight with Captured Spanish Arms, in: Chicago Tribune, 12.2.1925; ChTATOU, Aspectos, S. 69; NIMSCHOWSKI, Grundzüge, S. 267; YousSOUFI, Les institutions, S. 93f. Vgl. zur Organisations- 
In jeder Kabyle, die sich dem Rifverbund freiwillig oder unfreiwillig durch Unterwerfung anschloß, wurde ein Kontrollbeamter eingesetzt. Er war nur der Zentrale Ajdir gegenüber verpflichtet, seine Anordnungen mußten befolgt werden. Dies schränkte die Macht der lokalen Caïds wesentlich ein. Zur besseren Kontrolle der Kabylen diente ein Netz von Militärposten, denen zumeist Angehörige der Beni Ouriaghel vorstanden. Ein weitverzweigtes Telefonsystem wurde aufgebaut, auch um die Verwaltung der entfernteren Bezirke schneller gewährleisten zu können. Straßen wurden errichtet, eine reguläre Rifarmee aufgebaut. Zu den - geplatzten - Vorhaben gehörte sogar die Errichtung einer Staatsbank mit einer eigenen Währung, die »Riffan« genannt werden sollte ${ }^{124}$. Die Ansätze eines Schulsystems waren erkennbar ${ }^{125}$. Abdelkrim träumte von einer Universität in Ajdir, in der traditionelle und moderne Wissenschaften unter einem Dach wohnen sollten ${ }^{126}$.

\subsection{Primo de Riveras Staatsstreich und der spanische Verlust von Chichaouen}

In Spanien gab es derweil umwälzende Veränderungen. Mit einem Staatsstreich übernahm General Miguel Primo de Rivera y Orbaneja am 13. September 1923 mit Zustimmung des spanischen Königs die politische Macht. Marokko stellte für ihn ein schier unlösbares Problem dar. Seit 1909 hatte die spanische Einflußzone über zwei Milliarden Peseten gekostet und Tausende von Toten gefordert. Trotz des kolonialen Prestiges war sie nur ein Stiefkind spanischer Politik gewesen. Primo de Rivera und sein Militärdirektorium hatten zunächst drei Ziele für Marokko vor Augen - die Beendigung des RifKonflikts durch Verhandlungen, die Reduzierung der Kosten und der Truppen sowie die Reformierung und Stützung der Position der spanischen Armee.

struktur der "Rif-Republik« LOUTSKAÏA, À propos, S. 1-12. Abdelkrim bewunderte den Säkularismus der kemalistischen Reformen in der Türkei [Jacques FrÉMEAUX, La France et l'Islam depuis 1789, Paris 1991, S. 164; KHARCHICH, La France, S. 6; SHINAR, Abd alQadir, S. 167; MADARIAGA, España y el Rif, S. 564-566].

${ }^{124}$ PenNell, A Country, S. 129f., S. 141-143; MADARIAGA, España y el Rif, S. 569-571; ChTATOU, Aspectos, S. 68f.; KHARCHICH, La France, S. 96; YoussoufI, Les institutions, S. 93.

${ }^{125}$ Villain, Brun, Fellah ohne Faruk, S. 10; Pennell, A Country, S. 149f.; Ders., Morocco, S. 194; HART, The Aith Waryaghar, S. 389; NMSCHOWSKI, Grundzüge, S. 267.

${ }^{126}$ SPENCER PRYSE, Through the Lines, S. 465. 
Hochkommissar Luis Silvela y Casado mußte General Luis Aizpurú Mondéjar weichen. Schon im Dezember 1923 wurden 29000 Soldaten nach Spanien zurückgeschickt ${ }^{127}$.

Begleitet von neuerlichen Überfällen auf spanische Posten im Osten, weiteten die Rifkabylen ihren Einfluß bis zum Sommer 1924 auch nach Westen (Ghomara, Jebala) aus. Zum Teil betätigte sich Abdelkrim dort selbst als Eroberer, um unwillige Kabylen auf seine Seite zu bringen. Die von General Berenguer zwischen 1919 und 1922 eroberten Gebiete bis zur Oued-Lau-Linie waren durch Hunderte kleiner Blockhäuser und Militärposten gesichert worden. Dieses System - übernommen aus der Kolonialzeit in Kuba - erwies sich für das spanische Militär als strategischer Nachteil. Ein großer Teil der Truppen wurde für die Besetzung dieser Posten benötigt und war dort zur Tatenlosigkeit verurteilt. Ein weiterer großer Teil mußte die Versorgungskonvois für die Posten absichern. Dies führte zu einer gravierenden Unbeweglichkeit und Unflexibilität. Die Folge war, daß die Rifkämpfer ihre Kräfte ungefährdet an beliebigen Punkten bündeln konnten. So waren sie in der Lage, gezielte Militärschläge zu setzen oder die Forts zu umzingeln, um sie auszuhungern ${ }^{128}$. Den Guerillataktiken der Rifkabylen hatten die statischen spanischen Truppen außer ihrer Luftwaffe lange nichts entgegenzusetzen. Das bergige Gelände war wie geschaffen für Hinterhalte. Nachts gehörte es den Riftruppen, denn die Spanier zogen sich in der Regel in ihre Posten zurück. "Sobald es $5 \mathrm{Uhr}$ ist, hört der Spanier mit dem Gefecht auf«, wunderte sich der deutsche Fremdenlegionär Franz Sehring ${ }^{129}$.

Das spanische Militär versuchte, den Druck auf die Westfront mit verstärkten Luftbombardements im Raum Ajdir zu verringern. Trotzdem startete Abdelkrim im Juli 1924 seine größte Offensive seit 1921, die die Spanier im Westen völlig überraschte. Ein spanischer Posten nach dem anderen mußte aufgeben, bis Ende August die spanische West-Armee die Kontrolle völlig verloren hatte. Die Rifkämpfer kamen der heiligen Stadt Chichaouen, deren spanische Besetzung Abdelkrim beenden wollte, immer näher. Primo de Riveras 1924 verkündetes Programm der Halbabtretung Marokkos - damit war der Rückzug der spanischen Armee auf verstärkte Positionen gemeint - kam nun früher und anders als erwartet, denn Chichaouen war nicht mehr zu halten. Der Befehl zur Sammlung der Truppen des Westsektors in und um Chichaouen

${ }^{127}$ FLEMING, Primo de Rivera and Spain's Moroccan Problem, S. 85-88; PENNELL, A Country, S. 158f.; ÁlvareZ, The Betrothed of Death 2001, S. 91f.; PAYNE, Politics, S. 187; WOOLMaN, Rebels in the Rif, S. 121-123; BoYD, Praetorian Politics, S. 258-261; SUEIRO SEOANE, Spanish Colonialism, S. 48f.; SEPASGOSARIAN, Freundschaft, S. 20, S. 39-41.

${ }^{128}$ ASPREY, War in the Shadows, S. 271; FRUNSE, Zivilisatoren, S. 505f.; SHEEAN, War in Africa, S. 258.

${ }^{129}$ Franz SEHRING, Auf den Schlachtfeldern Marokkos. Die Leiden eines Deutschen in der spanischen Fremdenlegion. Erzählt nach eigenen Erlebnissen, Gumbinnen 1925, S. 26. Vgl. Hanns-Erich KAMINSKI, Spanisch-Marokko, in: Weltbühne 20 (1924) S. 718-720. 
kam am 25. September 1924. Rund 400 Posten und Blockhäuser wurden geräumt. Der Rückzug zur sogenannten Primo-de-Rivera-Linie zehn Kilometer südlich von Tetuan begann am 15. November 1924 und endete einen Monat später ${ }^{130}$.

7000 Rifkabylen und verbündete Jebalis, die sich im strömenden Regen der Winterzeit zumeist auf den Hügeln entlang der Straße von Chichaouen nach Tetuan postiert hatten, sorgten unter den nach Norden ziehenden spanischen Soldaten für panische Szenen, die denen von Annual ähnelten ${ }^{131}$. Es gab blutige Verluste mit Tausenden von Toten ${ }^{132}$. Am 17. November 1924 zog Oberbefehlshaber Si M'hammed mit den Riftruppen in Chichaouen ein. Es war ein erneuter Triumph für Abdelkrim. Im Jebala-Gebiet hatte sich bisher nur Ahmed el Risouni dem wachsenden Einfluß von Abdelkrim entgegenstellen können. Doch im Januar 1925 nahmen ihn Riftruppen gefangen. Abdelkrim, der nun etwa $75 \%$ der spanischen Einflußzone von Tetuan bis Temsamane kontrollierte, befand sich auf dem Höhepunkt seiner Macht. Außer Melilla, Tetuan, Ceuta, der Festung Nokour vor Alhucemas, der Straße von Tetuan nach Tanger und dem Gebiet im Westen der Einflußzone von Arzila bis Larache hatte Spanien alles verloren ${ }^{133}$.

${ }^{130}$ Álvarez, The Betrothed of Death 2001, S. 126-140; FleMng, Primo de Rivera and Abd-el-Krim, S. 141-150, S. 184-192, S. 202; DERS., Primo de Rivera and Spain's Moroccan Problem, S. 88f.; PAYNe, Politics, S. 212, S. 215; CHANDLER, Spain and her Moroccan Protectorate, S. 317f.; PENNELL, A Country, S. 168f.; WOOLMAN, Rebels in the Rif, S. 134 140; TOYNBEE, The Islamic World, S. 120 f; SUEIRO SEOANE, Spanish Colonialism, S. 53; José M. MARÍN ARCE, Primo de Rivera y la República del Rif durante los primeros años de la dictadura. Negociaciones con Abd-el-Krim en junio de 1925, in: RIPOLL PERELLó, LADERO QueSADA (Hg.), Actas, S. 271-286, S. 276-278; Ismael SAZ, Foreign Policy under the Dictatorship of Primo de Rivera, in: BALFOUR, PRESTON (Hg.), Spain, S. 53-72, S. 56.

${ }^{131}$ MOWRER, The House of Europe, S. 473; SPENCER PRYSE, Through the Lines, S. 443; PAYNe, Politics, S. 215; MLler, Frieden, S. 171; MEYER, Dreitausend Kilometer, S. 66, S. 68.

${ }^{132}$ Hinsichtlich der spanischen Verluste während des Rückzugs aus Chichaouen gibt es nur grobe Schätzungen: z.B. 1500 [FleMING, Primo de Rivera and Spain's Moroccan Problem, S. 89; SLavin, Anticolonialism, S. 50]; 2000 [PAYNe, Politics, S. 215]; 17000 [ASPREY, War in the Shadows, S. 272; FURNEAUX, Abdel Krim, S. 132; HARRIS, France, S. 147]; 14 000-15000 [PENNELL, A Country, S. 169]; über 20000 [FRUNSE, Zivilisatoren, S. 498]; 2000-18 000 [ÁLVAREZ, The Betrothed of Death 2001, S. 139]; 15000 [BALFOUR, Deadly Embrace, S. 104]; 17 000-20 000 [WOOLMAN, Rebels in the Rif, S. 140]; bis 20000 [KUNZ, MŨLLER, Giftgas, S. 114; NMSCHOWSK1, Grundzüge, S. 268].

${ }^{133}$ FLEMING, Primo de Rivera and Abd-el-Krim, S. 202f., S. 210f., S. 217; BaLFour, Deadly Embrace, S. 104f.; DAOUD, Abdelkrim, S. 201; MESA GUTIÉRREZ, Decada de sangre, S. 151; PAYNE, Politics, S. 217; HART, The Aith Waryaghar, S. 394; ÁlVAREZ, The Betrothed of Death 2001, S. 163f.; LA PORTE, La atracción del imán, S. 114f.; KHARCHICH, La France, S. 87; Pennell, A Country, S. 180; TOYNBEe, The Islamic World, S. 124; Usborne, The Conquest, S. 260; Kunz, Mūller, Giftgas, S. 116; CHARQL, Abdelkrim, S. 141-145. Risouni starb während seiner Gefangenschaft am 3. April 1925 in Tamasint [WOOLMAN, Rebels in the Rif, S. 163]. 


\subsection{Der Angriff der Rifkabylen auf Französisch-Marokko und Abdelkrims Kapitulation}

Während der ersten Jahre des Krieges hatte Frankreich eine neutrale Haltung der friedlichen Koexistenz gegenüber den Rifkabylen eingenommen, doch dann tauchten Anfang 1924 Rif-Kontingente am Nordufer des Ouergha-Flusses auf - direkt an der Nordgrenze des französischen Protektorats. Seit langer Zeit schon diente das fruchtbare Ouergha-Tal - der sogenannte »Brotkorb« der Rifkabylen - als Nachschubquelle für Lebensmittel ${ }^{134}$.

Generalresident Lyautey sah sich nun zu militärischen Gegenmaßnahmen gezwungen. Bislang waren französische Truppen noch nicht bis zur 1912 eher vage festgelegten Grenze zwischen der französischen und der spanischen Einflußzone in Marokko vorgedrungen. Nun überschritten sie im Mai und Anfang September 1924 den Ouergha-Fluß und etablierten 20 Kilometer nördlich eine neue Frontlinie auf dem Gebiet der Beni Zeroual. Aus französischer Sicht handelte es sich um eine Grenzbegradigung in Form einer rund 100 Kilometer langen Kette von Blockhäusern und Militärposten, die der Sicherung von Fes diente, und um eine >Polizei-Operation` zur Unterstützung des Alliierten Adelrahman Derkaoui gegen die Rifkabylen. Nun stand Abdelkrim vor einem Dilemma. Zwar wollte er keinen Ärger mit Frankreich. Andererseits betrachtete er das Ouergha-Tal als Teil der »Rif-Republik«. Seine Truppen erlitten hohe Verluste, als sie Anfang Juni 1924 zu kleineren Gegenschlägen ansetzten, um den eigenen Verbündeten innerhalb der Beni Zeroual zu helfen. Den Rest des Jahres konzentrierten sich die Aktivitäten von Abdelkrims Kämpfern auf den Westsektor der spanischen Zone. Eine trügerische Ruhe herrschte vor den französischen Grenzposten, die fast ein Jahr andauerte. Die Rifarmee befestigte ihrerseits ihren Teil der neuen Südfront und richtete in Targuist einen neuen Kommandoposten ein. Nach dem Sieg von Chichaouen wurden Truppen Ende 1924 in Richtung Süden geschickt ${ }^{135}$.

Rein rational sprach alles gegen einen Angriff der Rifkabylen auf das französische Protektorat. Sie waren zahlenmäßig unterlegen und hätten nun gegen gleich zwei Kolonialmächte an mehreren Fronten kämpfen müssen. Der landwirtschaftliche Reichtum des abgeschnittenen Ouergha-Tals war jedoch überlebenswichtig für das Rif, da dort die Ernten vom spanischen Giftgaseinsatz

${ }^{134}$ KUNZ, MÜLLER, Giftgas, S. 113; SLAVIN, Anticolonialism, S. 54.

${ }^{135}$ GerShOVICH, French Military Rule, S. 128f.; PENNELL, A Country, S. 182f., S. 185; TOYNBEE, The Islamic World, S. 128; KHARCHICH, La France, S. 347; HoISINGTON, Lyautey, S. 190-192; FRUNSE, Zivilisatoren, S. 511; WOOLMAN, Rebels in the Rif, S. 171; NIMSCHOwSKI, Grundzüge, S. 271; USBORNE, The Conquest, S. 264; René BONNET-DEVILLIERS, La Guerre du Rif. Ce que j'ai vu au Maroc, Paris 1926, S. 64f. 
weitgehend verseucht waren und eine Hungersnot drohte ${ }^{136}$. Es gab zudem verbindliche Allianzversprechen Abdelkrims für wichtige Teile der Beni Zeroual. Sollte die euphorische Stimmung nach den Siegen über die Spanier nicht abnehmen, benötigte er neue Erfolge. Hinter dem Angriff steckte auch die Hoffnung Abdelkrims, Frankreich zur Anerkennung der Unabhängigkeit des Rifstaates zu zwingen. Er verkalkulierte sich, denn er rechnete nicht damit, daß Frankreich und Spanien gegen ihn kooperieren würden. Abdelkrim machte seinen größten Fehler. Er befahl seinen Truppen, gegen Französisch-Marokko vorzurücken ${ }^{137}$.

Den französischen Militärs war bewußt, daß im Frühjahr 1925 ein Angriff zu befürchten war, und doch wurden sie von der Heftigkeit der Schläge völlig überrascht. Am 12. April 1925 begannen die rund 4000 bis 6000 Mann starken Riftruppen auf breiter Front den Vormarsch und durchbrachen die erste Verteidigungslinie französischer Posten auf der Nordseite der Ouergha. Bibane, der strategisch wichtigste Punkt vor Fes, ging für die Franzosen bis September 1925 verloren. Im Osten versuchten die Riftruppen, die Verbindung Fes-Taza-Oujda zu trennen, im Westen bedrohten sie Ouezzane. Im Zentrum des Angriffs waren sie im Juli 1925 nur noch 30 Kilometer von Fes entfernt und strebten einen Vorstoß ins Atlasgebirge an ${ }^{138}$. Die Lage der Franzosen war sehr emst. Marschall Lyautey befürchtete nicht nur den Verlust von Fes, sondern dachte auch über eine Evakuierung von Taza nach. Erst Verstärkungen aus Algerien und aus Europa stabilisierten die Lage ${ }^{139}$.

Angesichts der militärischen Situation war Lyautey zur Kooperation mit Spanien bereit. Sein Dreifach-Plan zur Bekämpfung der »Abd-el-Kriminellen« - wie die Rifkämpfer von der kolonialen Lobby Frankreichs genannt wurden sah eine Blockade der Rifküste, massive Luft-Bombardements und einen direkten Angriff auf Ajdir über eine Landung in der Bucht von Alhucemas vor.

\footnotetext{
${ }^{136} \mathrm{KUNZ}$, »Con ayuda《, S. 180.

${ }^{137}$ Balfour, Deadly Embrace, S. 106; PenNel, A Country, S. 182-185; FurNeAuX, Abdel Krim, S. 146f.; KUNZ, MŪLlER, Giftgas, S. 116, S. 120; GERSHOVICH, French Military Rule, S. 129; Wollman, Rebels in the Rif, S. 172; FleMing, Primo de Rivera and Abd-elKrim, S. 229; KHARCHICH, Observations, S. 230; USBORNE, The Conquest, S. 30; HARRIS, France, S. 199.

${ }^{138}$ GERSHOVICH, French Military Rule, S. 130-133; BALFOUR, Deadly Embrace, S. 108; Douglas PORCH, The French Foreign Legion. A Complete History of the Legendary Fighting Force, New York 1991, S. 399; Anthony ClaYTON, France, Soldiers and Africa, London u.a. 1988, S. 108f.; TOYNBEE, The Islamic World, S. 137f.; WoOLMAN, Rebels in the Rif, S. 174-179; HoISINGTON, Lyautey, S. 193-200; FlEMNG, Primo de Rivera and Abd-elKrim, S. 234-236; FRUNSE, Zivilisatoren, S. 513-518. Eine detaillierte Beschreibung der Kämpfe in: USBORNE, The Conquest, S. 269-285; RIVET, Lyautey, Bd. 3, S. 282-296.

${ }^{139}$ PENNELl, A Country, S. 190; WoOLMAN, Rebels in the Rif, S. 183; DAOUD, Abdelkrim, S. 248; TOYNBEE, The Islamic World, S. 149; KUNZ, MÜller, Giftgas, S. 133; FréMEAUX, La France, S. 159; Alfred ZAEPER, Unter der Glutsonne Marokkos gegen Abd el Krim, Leipzig 1932, S. 23.
} 
Dies war nur zusammen mit den Spaniern möglich. Am 18. Juni 1925 trafen sich französische und spanische Delegationen erstmals in Madrid, um ein gemeinsames Vorgehen gegen Abdelkrim und eine abschließende Lösung des Rifproblems zu besprechen. Verschiedene Abkommen betrafen Vorkehrungen gegen Waffenschmuggel, Prinzipien für - getrennte und daher vergebliche Friedensverhandlungen und zuletzt am 25. Juli 1925 eine gemeinsame Militärstrategie $^{140}$.

Trotzdem war die französische Regierung mit Marschall Lyautey unzufrieden. Er mußte im Juli 1925 das Oberkommando über die französischen Truppen Marokkos an General Naulin abgeben. An dessen Seite trat Generalissimus Marschall Philippe Pétain. Lyautey legte zwei Wochen später sein Amt als Generalresident nieder und kehrte nach Frankreich heim. Sein Nachfolger wurde der bisherige Generalgouverneur von Algerien Théodore Steeg ${ }^{141}$.

Der September 1925 brachte die Wende im Rifkrieg. Spanien hatte mittlerweile seine Truppenstärke auf 200000 Mann erhöht. An der Nordgrenze des französischen Protektorats standen rund 160000 Mann gegen etwa 60000 bis 80000 Rifkämpfer. Den Franzosen gelang es, Bibane zurückzuerobern und die Rifarmee zwischen dem 11. und dem 15. September 1925 aus FranzösischMarokko zu verdrängen. Die Zielrichtung ging weiter nach Norden - dies hatten Spanien und Frankreich im Juli in Madrid vereinbart ${ }^{142}$. Pétain führte in Marokko europäische Kriegstechniken mit schnellen, von der Luftwaffe unterstützten Offensiven ein. Sein Ziel war das Herz der »Rif-Republik« - das Gebiet der Beni Ouriaghel, die Heimat Abdelkrims ${ }^{143}$.

${ }^{140}$ HUETZ DE LEMPS, La collaboration, S. 92f.; RIVET, Lyautey, Bd. 3, S. 295; HoISINGTON, Lyautey, S. 199; Rom LANDAU, Moroccan Drama, 1900-1955, London 1956, S. 125; PENNELL, A Country, S. 196f.; Javier TUSELL, La dictadura de Primo de Rivera 1923-1930, in: José M. JOVER ZAMORA (Hg.), La España de Alfonso XIII. El estado y la política 19021931, Bd. 2: Del plano inclinado hacia la dictadura al final de la monarquía 1922-1931, Madrid 1995 (Historia de España, Menéndez Pidal, 38), S. 131-747, S. 316f.; SUEIRO SEOANE, España, S. 205-209; KHARCHICH, La allianza, S. 74-77; WOOLMAN, Rebels in the Rif, S. 179-181; MARín ARCE, Primo de Rivera, S. 282; TOYNBEE, The Islamic World, S. 140144.

${ }^{141}$ GerShoviCH, French Military Rule, S. 137; RIVET, Lyautey, Bd. 3, S. 296-310; HoISINGTON, Lyautey, S. 201-203; Pierre BOURGET, La rivalité Pétain-Lyautey de 1925 au Maroc. Un nouvel éclairage, in: Guerres mondiales et conflits contemporains 46 (1996) S. 125133; Barnett SINGER, Lyautey. An Interpretation of the Man and the French Imperialism, in: Journal of Contemporary History 26 (1991) S. 131-157, S. 147; WoOLMAN, Rebels in the Rif, S. 183, 194f.; FrÉMEAUX, La France, S. 159; TOYNBEE, The Islamic World, S. 150; USBORNE, The Conquest, S. 288-296; Nicholas ATKIN, Pétain, London 1998, S. 48-51.

${ }_{142}$ WOOLMAN, Rebels in the Rif, S. 186f.; FrémEAUX, La France, S. 160; PORCH, Foreign Legion, S. 405.

${ }^{143}$ HUETZ DE LEMPS, La collaboration, S. 98; PAYNE, Politics, S. 221; TOYNBEE, The Islamic World, S. 146. 
Im Norden schlug Spanien zu und eröffnete zusätzlich zum Süden, Westen und Osten eine vierte Front für die Rifkabylen. Am 8. September 1925 gab Primo de Rivera trotz schlechter Witterungsverhältnisse den Befehl zur Landung bei Cebadilla westlich der Bucht von Alhucemas, wo 36 spanische Kriegsschiffe und 63 Truppentransporter aufgeboten waren. Trotz harter Gegenwehr der Rifarmee fiel Ajdir am 2. Oktober 1925 in die Hände der Spanier. Der Verlust der Hauptstadt bedeutete den Anfang vom Ende der »Rif-Republik«. Neun Tage später zerstörten spanische Truppen Aït Kamara, das Heimatdorf Abdelkrims. Der Regierungssitz der Rifkabylen wurde nach Tamasint verlegt. In Targuist wurde das neue Hauptquartier der Rifarmee eingerichtet. Nur der einbrechende Winter mit seinen heftigen Regenfällen bewahrte die Rifkabylen vor weiteren Gebietseinbußen ${ }^{144}$.

Dennoch war Ende 1925 noch keine militärische Entscheidung gefallen. Die geplante Vereinigung der französischen und spanischen Truppen auf der Linie Taza-Ajdir, die das Reich Abdelkrims in zwei Teile spalten sollte, war nicht geglückt. Die meisten Kabylen folgten Abdelkrim weiterhin, auch wenn es im Westen bei den Jebala gärte. Die militärische Organisation war noch intakt ${ }^{145}$. Abdelkrim wußte zwar, daß er den Krieg nicht gewinnen konnte, aber er hoffte, daß Frankreich mit dem Erreichten zufrieden war und mit ihm über einen Separatfrieden verhandeln würde. Doch als Aristide Briand im November 1925 die Regierung übernahm, wurde schnell deutlich, daß er auf eine militärische Lösung setzte ${ }^{146}$. Die Friedenskonferenz von Oujda von April bis Mai 1926 war von vornherein zum Scheitern verurteilt. Die Delegierten aus dem Rif, Spanien und Frankreich führten die Gespräche in einer Atmosphäre gegenseitigen Mißtrauens. Die Rif-Emissäre weigerten sich, über eine sofortige Freilassung der Kriegsgefangenen vor dem Ende der Feindseligkeiten zu reden. Sie wollten sie als Trumpfkarte behalten. Daher platzte die Konferenz am 7. Mai $1926^{147}$.

Der zuvor im Februar 1926 von Marschall Pétain und General Primo de Rivera vereinbarte gemeinsame Angriffsplan wurde nun umgehend umgesetzt. Von allen Seiten rückten spanische und französische Truppen vor - zusammen rund 500000 Mann $^{148}$. Sie stießen, von wenigen Ausnahmen abgesehen, kaum noch auf nennenswerten Widerstand. Das Scheitern der Oujda-Konferenz hat-

144 Fleming, Primo de Rivera and Abd-el-Krim, S. 298-300; PenNELL, Morocco since 1830, S. 191; DERS., A Country, S. 198f., S. 205; TUSELL, La dictadura, S. 327-330; KUNZ, MülLER, Giftgas, S. 162-167; ÁlVAREZ, The Betrothed of Death 2001, S. 168-178; SUERO SEOANE, España, S. 274-277; MESA GuTtÉRREZ, Decada de sangre, S. 154f.; WOOLMAN, Rebels in the Rif, S. 187-192; PAYNe, Politics, S. 220; HILLS, Franco, S. $139 f$.

${ }^{145}$ TOYNBEe, The Islamic World, S. 147f.; Fleming, Primo de Rivera and Abd-el-Krim, S. $327 \mathrm{f}$.

${ }^{146}$ HART, The Aith Waryaghar, S. 398.

${ }^{147}$ WOOLMAN, Rebels in the Rif, S. 199-204; PENNELL, A Country, S. 212-214.

${ }^{148}$ BoichUT, Campagne, S. 314; USBORNE, The Conquest, S. 301; PAYNE, Politics, S. 221. 
te die Moral im Rif gebrochen. Eine auf Tetuan gerichtete Gegenoffensive der Rifkabylen verpuffte rasch. Am 18. Mai 1926 eroberten die Spanier den Ausgangspunkt des Rifkrieges - Annual - zurück. Zwei Tage später gelang die Verbindung mit französischen Kontingenten an der Ajdir-Front. Targuist fiel am 23. Mai 1926. Vier Tage später kapitulierte Abdelkrim und ergab sich mit seiner Familie den Franzosen. Der Rifkrieg war vorbei, auch wenn Spanien noch bis Juli 1927 brauchte, um die eigene Einflußzone endgültig zu unterwerfen ${ }^{149}$.

Abdelkrim trat noch 1926 sein Exil auf der Insel La Réunion im Indischen Ozean an. Als er 1947 nach Frankreich zurückgebracht werden sollte, konnte er während der Fahrt durch den Suez-Kanal entkommen. Als Präsident des Comité de libération du Maghreb engagierte er sich in Kairo bis zu seinem Tod im Jahre 1963 für die Befreiungsbewegungen Nordwest-Afrikas ${ }^{150}$. Der "Vercingetorix des Rifs ${ }^{151}$ kehrte nie in seine Heimat zurück.

${ }^{149}$ HART, The Aith Waryaghar, S. 399-401; Balfour, Deadly Embrace, S. 116f.; ÁlVAREZ, The Betrothed of Death 2001, S. 194-204; PENNELL, A Country, S. 215-218; MESA GUTIÉRREZ, Decada de sangre, S. 159f.; DAOUD, Abdelkrim, S. 345f.; KUNZ, MÜLLER, Giftgas, S. 171f.; PaYNe, Politics, S. 222; Woolman, Rebels in the Rif, S. 204-206, S. 213f.; Pierre Khorat, La fin d'Abd-el-Krim, in: Correspondant 304 (1926) S. 321-357. Sporadische Widerstandskämpfe im Rif gab es noch bis 1934 [ASPREY, War in the Shadows, S. 275]. Der letzte große Aufstand im Rif - diesmal gegen den marokkanischen König - dauerte von Oktober 1958 bis Februar 1959. In einem 18-Punkte-Programm wurde unter anderem auch die Rückkehr Abdelkrims gefordert. Mit 20000 Mann schlug Kronprinz Hassan die Rebellion blutig nieder [PENNELL, Morocco since 1830, S. 304; HART, The Aith Waryaghar, S. 428f.; HART, Tribe and Society, S. 90-95; WOOLMAN, Rebels in the Rif, S. 227f.]. ${ }_{150}$ PENNELL, A Country, S. 216; DERS., Morocco, S. 278; CHARQI, Abdelkrim, S. 169-182; KUNZ, MÜLLER, Giftgas, S. 173; MìGE, Abd el-Krim, S. 75f.; HART, The Aith Waryaghar, S. 400f.; WOOLMAN, Rebels in the Rif, S. 207f., S. 223. Zum Exil und zur Kairoer Zeit Abdelkrims: DAOUD, Abdelkrim, S. 349-387; MONTAGNE, Abd el Krim, S. 314-322.

${ }^{151}$ HoISINGTON, Lyautey, S. 189; MONTAGNE, Abd el Krim, S. 304; Carl von OSSIETZKY, Abd el Krim, Rif und Riffe, in: Weltbühne 22 (1926) S. 833-837, S. 833. 
